\title{
Water vapor emission from IRC+10216 and other carbon-rich stars: model predictions and prospects for multitransition observations
}

\author{
Eduardo González-Alfonso \\ Universidad de Alcalá de Henares, Departamento de Física, Campus Universitario, \\ E-28871 Alcalá de Henares, Madrid, Spain \\ eduardo.gonzalez@uah.es \\ David A. Neufeld \\ Department of Physics \& Astronomy, The Johns Hopkins University, 3400 N. Charles \\ Street, Baltimore, Maryland 21218 \\ and \\ Gary J. Melnick \\ Harvard-Smithsonian Center for Astrophysics, 60 Garden Street, Cambridge, MA 02138
}

\begin{abstract}
We have modeled the emission of $\mathrm{H}_{2} \mathrm{O}$ rotational lines from the extreme C-rich star IRC+10216. Our treatment of the excitation of $\mathrm{H}_{2} \mathrm{O}$ emissions takes into account the excitation of $\mathrm{H}_{2} \mathrm{O}$ both through collisions, and through the pumping of the $\nu_{2}$ and $\nu_{3}$ vibrational states by dust emission and subsequent decay to the ground state. Regardless of the spatial distribution of the water molecules, the $\mathrm{H}_{2} \mathrm{O} 1_{10}-1_{01}$ line at $557 \mathrm{GHz}$ observed by the Submillimeter Wave Astronomy Satellite (SWAS) is found to be pumped primarily through the absorption of dust-emitted photons at $6 \mu \mathrm{m}$ in the $\nu_{2}$ band. As noted by previous authors, the inclusion of radiative pumping lowers the ortho- $\mathrm{H}_{2} \mathrm{O}$ abundance required to account for the $557 \mathrm{GHz}$ emission, which is found to be $(0.5-1) \times 10^{-7}$ if the presence of $\mathrm{H}_{2} \mathrm{O}$ is a consequence of vaporization of orbiting comets or Fischer-Tropsch catalysis. Predictions for other submillimeter $\mathrm{H}_{2} \mathrm{O}$ lines that can be observed by the Herschel Space Observatory (HSO) are reported. Multitransition HSO observations promise to reveal the spatial distribution of the circumstellar water vapor, discriminating among the several hypotheses that have been proposed for the origin of the $\mathrm{H}_{2} \mathrm{O}$ vapor in the envelope of $\mathrm{IRC}+10216$. We also show that, for observations with $\mathrm{HSO}$, the $\mathrm{H}_{2} \mathrm{O} 1_{10}-1_{01} 557 \mathrm{GHz}$ line affords the greatest sensitivity in searching for $\mathrm{H}_{2} \mathrm{O}$ in other C-rich AGB stars.
\end{abstract}


Subject headings: Stars: abundances — Stars: AGB and post-AGB — stars: individual (IRC+10216) — ISM: molecules — radiative transfer — submillimeter

\section{Introduction}

The discovery of water vapor emission in the extreme C-rich AGB star IRC+10216 with SWAS (Melnick et al. 2001), its confirmation by ODIN (Hasegawa et al. 2006), and the subsequent detection of other O-bearing molecules like OH (Ford et al. 2003), $\mathrm{H}_{2} \mathrm{CO}$ (Ford et al. 2004), and $\mathrm{C}_{3} \mathrm{O}$ (Tenenbaum et al. 2006), have challenged our current understanding of the chemistry in envelopes around C-rich AGB stars. According to standard models, essentially all oxygen nuclei are predicted to be locked into $\mathrm{CO}$ or $\mathrm{SiO}$ with no reservoir to form other O-bearing molecules (except for low abundances of species such as $\mathrm{HCO}^{+}$in the outer envelope where photochemistry is important). Therefore, the unexpectedly high abundances found for $\mathrm{H}_{2} \mathrm{O}, \mathrm{OH}$, and $\mathrm{H}_{2} \mathrm{CO}$, indicate that several processes not included in standard models for C-rich environments are driving the oxygen chemistry, but the dominant water production mechanism is still a source of debate.

Four distinct mechanisms have been considered as possible sources of the observed water vapor in $\mathrm{IRC}+10216$, each one making a specific prediction for the $\mathrm{H}_{2} \mathrm{O}$ spatial distribution in the envelope: $(i)$ chemistry in the inner envelope, which would imply the presence of $\mathrm{H}_{2} \mathrm{O}$ in the warmest and densest regions; $(i i)$ vaporization of icy orbiting bodies that have survived from the main sequence into the C-rich AGB phase (Melnick et al. 2001; Ford \& Neufeld 2001), predicting the release of $\mathrm{H}_{2} \mathrm{O}$ at intermediate radii of $R_{\text {int }}=(1-5) \times 10^{15} \mathrm{~cm}$; (iii) grain surface reactions, such as the Fischer-Tropsch catalysis on the surfaces of small metallic grains (Willacy 2004), which predicts $\mathrm{H}_{2} \mathrm{O}$ to attain a nearly uniform abundance at radii larger than $R_{\text {int }}=(1.5-2) \times 10^{15} \mathrm{~cm} ;(i v)$ chemistry involving photodissociation products in the outermost regions of the envelope: a specific mechanism relying on the radiative association $\mathrm{O}+\mathrm{H}_{2} \rightarrow \mathrm{H}_{2} \mathrm{O}+\gamma$ has been proposed by Agúndez \& Cernicharo (2006, hereafter AC06), and predicts $\mathrm{H}_{2} \mathrm{O}$ to be present at radii higher than $R_{\text {int }} \approx 4 \times 10^{16} \mathrm{~cm}$. In all cases, $\mathrm{H}_{2} \mathrm{O}$ is expected to have a uniform abundance from $R_{\text {int }}$ up to the external region where it is photodissociated producing $\mathrm{OH}$.

The only water line detected so far in IRC +10216 , the ortho- $\mathrm{H}_{2} \mathrm{O} 110101$ transition at $557 \mathrm{GHz}$, is the ground-state transition, with the upper level at only $27 \mathrm{~K}$ above the ground rotational level, and cannot discriminate -by itself- between the processes listed above. Nevertheless, the launch of the Herschel Space Observatory (HSO) will allow us to observe other $\mathrm{H}_{2} \mathrm{O}$ lines in the submillimeter and far-infrared domains, permitting us to infer the region where the $\mathrm{H}_{2} \mathrm{O}$ emission arises, and will potentially favor one of the 
proposed formation mechanisms. The Heterodyne Instrument for the Far Infrared (HIFI) onboard HSO will provide very high spectral resolution observations (0.14-1.0 MHz), thus permitting the lines to be velocity resolved. In this paper, we model the $\mathrm{H}_{2} \mathrm{O}$ emission from IRC +10216 to show how the $\mathrm{H}_{2} \mathrm{O}$ spatial distribution can be inferred from HSO multitransition observations. Also, we explore which $\mathrm{H}_{2} \mathrm{O}$ transition provides the most sensitive means of searching for water vapor around AGB stars other than IRC+10216; such a search would determine whether the occurence of $\mathrm{H}_{2} \mathrm{O}$ in $\mathrm{C}$-rich environments is widespread.

\section{Model description}

Our model for IRC +10216 assumes a stellar mass loss rate of $\dot{M}=3 \times 10^{-5} \mathrm{M}_{\odot} \mathrm{yr}^{-1}$. The gas velocity field, turbulent velocity, and $\mathrm{H}_{2}$ density and gas temperature profiles are those adopted by Melnick et al. (2001) (see references therein), and listed in Table 1. A distance to the source $D=170 \mathrm{pc}$ is assumed.

The adopted gas temperature profile is an approximate fit (e.g. Glassgold 1996) to the results obtained by Kwan \& Linke (1982), who calculated the thermal profile through the circumstellar envelope of IRC+10216 from the energetic balance between gas heating and cooling. These calculations included gas-dust collisional heating and both adiabatic and CO line cooling. HCN line cooling was ignored and, subsequently, the ISO far-infrared spectrum of IRC+10216 showed strong HCN rotational emission from both the ground and excited vibrational states (Cernicharo et al. 1996). Nevertheless, radiative transfer calculations appear to indicate that HCN radiative excitation through absorption of infrared photons emitted by warm dust dominates over collisional excitation (González-Alfonso \& Cernicharo 1999a; Dinh-V-Trung \& Nguyen-Q-Rieu 2000), and in such a case the observed HCN emission would not involve any further cooling.

It is shown below that the continuum emission provides the dominant excitation source for all $\mathrm{H}_{2} \mathrm{O}$ lines of interest, mainly through absorption of $6 \mu \mathrm{m}$ photons in the $\nu_{2}$ band and subsequent decay, and so a model for the dust emission from IRC+10216 is required. We have used a radiative transfer code to compute, from the balance between heating and cooling at any radial position in the envelope, the dust temperature profile (González-Alfonso \& Cernicharo $1999 b)$. Our model simulates the dust properties by making use of an empirical spectral in$\operatorname{dex} \beta$. The inferred dust parameters are listed in Table 1, and the goodness of the fit, shown in Fig. 1, ensures that the continuum radiation density is well reproduced at least at $r \gtrsim 2 \times 10^{15} \mathrm{~cm}$, where it varies as $\sim r^{-2}$.

The radiative transfer code used to determine the $\mathrm{H}_{2} \mathrm{O}$ excitation and emission has 
been described in González-Alfonso \& Cernicharo (1997, 1999b). The calculations assume spherical symmetry, and the envelope is divided into 60 spherical shells to account for the variations in the physical and chemical conditions with distance to the star. The dust and $\mathrm{H}_{2} \mathrm{O}$ are assumed to be coexistent within any shell, and the non-local radiative transfer is simulated through rays that cross the source. The code first computes the $\mathrm{H}_{2} \mathrm{O}$ statistical equilibrium populations at any radial position in the envelope with the use of an iterative procedure based on an approximate Newton-Raphson operator (Schönberg \& Kempe 1986). A maximum relative variation of populations of $10^{-3}$ was established as the convergence criterion; a much tighter criterion was found in some test models to change line fluxes by at most $0.2 \%$. Once convergence is achieved, the resulting line profiles and fluxes are calculated. We report the expected intensities in units of main-beam temperature $\left(T_{M B}\right)$, with a halfpower beam width of HPBW $=240^{\prime \prime}$ to simulate SWAS measurements, and HPBW ranging from 12..5 (1703-1910 GHz) to 41" (480-640 GHz) for HSO-HIFI observations (see Table 3 , below).

Spectroscopic data for $\mathrm{H}_{2} \mathrm{O}$ were taken from the Hitran 2004 database (Rothman et al. 2005). We included the ground and the $\nu_{2}=1$ and $\nu_{3}=1$ excited vibrational states, but the $\nu_{3}=1$ state was found to have a negligible effect on the emission from the ground state lines. The $\nu_{1}=1$ state was ignored because the $\nu_{1}$ band is much weaker than the $\nu_{3}$ band, and more excited vibrational levels are also ignorable because they are weakly connected to the ground vibrational state and/or the continuum emission from IRC +10216 weakens in the near infrared. Up to 40 rotational levels per vibrational state for both ortho- $\mathrm{H}_{2} \mathrm{O}$ and para- $\mathrm{H}_{2} \mathrm{O}$ were taken into account, but the code allows this number to be decreased in external regions with low excitation, with the aim of ensuring and accelerating convergence.

The calculations simulate both collisional excitation among rotational levels of the ground vibrational state, and radiative excitation in all transitions. Vibrational excitation through collisions is neglected. Collisional rates among the 10 lowest rotational levels for $T_{k} \leq 140 \mathrm{~K}$ are taken from Phillips, Malluendes \& Green (1996). We extrapolate these rates to higher $T_{k}$ by multiplying the excitation rates for $T_{k}=140 \mathrm{~K}$ by $\exp \left\{\Delta E\left(1 / 140-1 / T_{k}\right)\right\}$, where $\Delta E(K)=E_{\text {up }}-E_{\text {low }}$ is the energy difference between the upper and lower levels. The application of this strategy appears to be justified, since the use of the Phillips et al. (1996) collisional rates at $T_{k}=140 \mathrm{~K}$, together with the quoted extrapolation, yield collisional rates for $T_{k}=20-120 \mathrm{~K}$ that reproduce the values given by Phillips et al. (1996) within a factor of 1.6. Also, some estimate of the collisional rates among levels not considered by Phillips et al. (1996) is required to simulate the collisional excitation in the innermost regions of the envelope. With this aim, we have found that if the $\mathrm{H}_{2} \mathrm{O}-\mathrm{He}$ collisional excitation rates for the 10 lowest rotational levels given by Green, Maluendes, \& McLean (1993), are multiplied by a global factor of 5 , most of the $\mathrm{H}_{2} \mathrm{O}-\mathrm{H}_{2} J=1$ rates given by Phillips et al. 
(1996) are reproduced within a factor of 2.5; similarly, most of the $\mathrm{H}_{2} \mathrm{O}-\mathrm{H}_{2} \mathrm{~J}=0$ rates given by Phillips et al. (1996) are reproduced within the same factor if the Green et al. rates are multiplied by a factor of 1.35, the difference in reduced mass when $\mathrm{H}_{2}$ is the collision partner rather than He. After applying these corrections to the Green et al. (1993) coefficients, some individual rates are still strongly underestimated, by 1-2 orders of magnitude, relative to the Phillips et al. (1996) values, specially in the case of collisions between para- $\mathrm{H}_{2} \mathrm{O} 0_{00}$ and $\mathrm{H}_{2} J=1$ (see discussion in Phillips et al. 1996). Nevertheless, the collisional excitation of a high-energy level in high-density and high-temperature regions is the result of collisional pumping from various other levels, and so individual discrepancies are to some extent diluted. In summary, we have used the Green et al. (1993) excitation rates, multiplied by the factors quoted above, to simulate the collisional excitation among levels not considered in Phillips et al. (1996). The adopted extrapolation does not critically affect results because, as shown below, radiative excitation dominates over collisional excitation in all lines of interest.

We have generated a set of models that differ only in the inner radius of the $\mathrm{H}_{2} \mathrm{O}$ shell, $R_{\text {int }}$, and in the $\mathrm{H}_{2} \mathrm{O}$ abundance, $X\left(\mathrm{H}_{2} \mathrm{O}\right)$. The different values of $R_{\text {int }}$ account for different spatial distributions of $\mathrm{H}_{2} \mathrm{O}$ in the envelope of $\mathrm{IRC}+10216$, and $X\left(\mathrm{H}_{2} \mathrm{O}\right)$ is derived in such a way that the predicted flux of the $\mathrm{o}-\mathrm{H}_{2} \mathrm{O} 110101$ is $\approx 10^{-20} \mathrm{~W} \mathrm{~cm}^{-2}$ as observed by SWAS (Melnick et al. 2001). The ortho-to-para $\mathrm{H}_{2} \mathrm{O}$ abundance ratio is assumed to be $3: 1$. In all models, we have adopted an external radius of the $\mathrm{H}_{2} \mathrm{O}$ shell $R_{\text {out }}=4 \times 10^{17} \mathrm{~cm}$, where $\mathrm{H}_{2} \mathrm{O}$ is assumed to be photodissociated. This value of $R_{\text {out }}$ is similar to that obtained by Willacy (2004) and AC06 through photodissociation models of the external shells of the IRC+10216 circumstellar envelope. The photodissociation radius depends on several parameters and could be significantly lower than the quoted value (Ford et al. 2003; Netzer \& Knapp 1987), but test models indicate that line flux ratios for $R_{\text {int }} \lesssim 8 \times 10^{15} \mathrm{~cm}$ vary by less than $20 \%$ if $R_{\text {out }}$ is decreased to $5 \times 10^{16} \mathrm{~cm}$. For high values of $R_{\text {int }}$, we give in section 4.3 an analytic expression for the expected flux of the o- $\mathrm{H}_{2} \mathrm{O} 110101$ line as a function of both $R_{\text {int }}$ and $R_{\text {out }}$, showing that results are not very sensitive to $R_{\text {out }}$ as long as $R_{\text {out }} / R_{\text {int }} \gg 1$.

We have selected three of those models, called $A, B$, and $C$, that correspond to the proposed formation mechanisms described in section 1. The adopted values of $R_{\text {int }}$ and the inferred ortho- $\mathrm{H}_{2} \mathrm{O}$ abundance, $X\left(\mathrm{o}-\mathrm{H}_{2} \mathrm{O}\right)$, are listed in Table 2, Model $A$ simulates $\mathrm{H}_{2} \mathrm{O}$ originating in the innermost regions of the envelope. Model $B$ applies to both the cometary hypothesis and the Fischer-Tropsch catalysis mechanism. Vaporization of icy bodies predicts that $\mathrm{H}_{2} \mathrm{O}$ is released at a radius of $(1-5) \times 10^{15} \mathrm{~cm}$, depending on the evolutionary stage of the star after the onset of the TP-AGB phase (Ford \& Neufeld 2001). The formation of $\mathrm{H}_{2} \mathrm{O}$ due to Fischer-Tropsch catalysis on the surfaces of iron grains predicts that the $\mathrm{H}_{2} \mathrm{O}$ abundance attains its maximum value at $(1.5-2) \times 10^{15} \mathrm{~cm}$ (Willacy 2004). Therefore, these two models predict similar $\mathrm{H}_{2} \mathrm{O}$ spatial distributions, although the Fischer-Tropsch 
mechanism appears to be more restrictive. Model $C$ assumes $\mathrm{H}_{2} \mathrm{O}$ to be generated as a consequence of photodissociation in the outermost regions of the envelope (AC06).

\section{Model results}

\subsection{The $\mathbf{H}_{2} \mathrm{O} 1_{10}-1_{01}$ line in $\mathrm{IRC}+\mathbf{1 0 2 1 6}$}

Figure 2 compares the SWAS 110101 ortho- $\mathrm{H}_{2} \mathrm{O}$ continuum-subtracted spectrum (from Melnick et al. 2001) with the line profiles obtained for models $A, B$, and $C$, with the SWAS beamsize. All models yield similar line profiles because in all three cases the emission region is small compared to the SWAS $4^{\prime}$ beam. The line shapes obtained with the smaller ODIN $2^{\prime}$ beam (not shown) are also similar because, even in model $C, 70 \%$ of the emission arises within a spherical shell of diameter $1^{\prime} .5\left(2.3 \times 10^{17} \mathrm{~cm}\right)$, which is significantly smaller than the ODIN beamsize. The $\mathrm{o}-\mathrm{H}_{2} \mathrm{O}$ abundance (relative to $\mathrm{H}_{2}$ ) required to account for the observed flux, is plotted in Fig. 3 as a function of $R_{\text {int }}$; it increases slowly for low $R_{\text {int }}$ because the innermost regions of the envelope contribute little to the emission at $557 \mathrm{GHz}$. For $R_{\text {int }}=2 \times 10^{15} \mathrm{~cm}($ model $B)$, the required ortho- $\mathrm{H}_{2} \mathrm{O}$ abundance, $X\left(\mathrm{o}-\mathrm{H}_{2} \mathrm{O}\right)=5.4 \times 10^{-8}$ (Table 2), is one order of magnitude lower than that reported previously by Melnick et al. (2001) and Hasegawa et al. (2006). As first noted by AC06 for the case of high $R_{\text {int }}$, this result is a consequence of the pumping of the $\nu_{2}=1$ vibrational state by dust emission at 6 $\mu \mathrm{m}$ and the subsequent decay to the ground state, which dominates the excitation of $\mathrm{H}_{2} \mathrm{O}$ in our models. In the case of the $\mathrm{o}^{-} \mathrm{H}_{2} \mathrm{O} 557 \mathrm{GHz}$ line, the importance of mid-IR pumping can be shown as follows. The radiative path that dominates the pump of the $1_{10}$ rotational level is $1_{01} \rightarrow \nu_{2} 1_{10} \rightarrow 2_{21} \rightarrow 1_{10}$. The associated pumping rate per molecule in the $1_{01}$ level is given by $\Gamma_{r}=B_{l u}\langle J\rangle \eta_{1} \eta_{2}$, where $B_{l u}$ is the Einstein coefficient for absorption of radiation in the $1_{01} \rightarrow \nu_{2} 1_{10}$ line, $\langle J\rangle$ is the continuum intensity at $6.2 \mu \mathrm{m}$ averaged over all angles and integrated over the line profile, $\eta_{1}$ is the fraction of molecules in $\nu_{2} 1_{10}$ that decay to $2_{21}$, and $\eta_{2}$ is the fraction of molecules in $2_{21}$ that decay to $1_{10}$. Ignoring line opacity effects, and estimating $\langle J\rangle$ as

$$
\langle J\rangle=\frac{D^{2}}{4 \pi r^{2}} F_{6.2 \mu \mathrm{m}}
$$

where $F_{6.2 \mu \mathrm{m}}=2.5 \times 10^{-13} \mathrm{~W} \mathrm{~cm}^{-2} \mu \mathrm{m}^{-1}$ is the observed $6.2 \mu \mathrm{m}$ continuum flux, and $r$ is the distance from the star, one obtains

$$
\Gamma_{r} \approx \frac{0.02}{r_{15}^{2}} \mathrm{~s}^{-1},
$$

where $r_{15}=r /\left(10^{15} \mathrm{~cm}\right)$. The ro-vibrational lines are indeed optically thin in all our models. The $1_{01} \rightarrow \nu_{2} 1_{10}$ and $1_{01} \rightarrow \nu_{2} 2_{12}$ have similar opacities; for $R_{\text {int }} \gtrsim 2 \times 10^{16} \mathrm{~cm}$, essentially 
all o- $\mathrm{H}_{2} \mathrm{O}$ molecules are in the ground $1_{01}$ level (Fig. $4 \mathrm{a}$ ) and the radial opacities of the quoted lines are given by

$$
\begin{aligned}
\tau_{r} & \approx 3 \times 10^{-2} \times\left(\frac{0.65 \mathrm{~km} \mathrm{~s}^{-1}}{\sigma_{v}}\right) \times\left(\frac{X\left(\mathrm{o}-\mathrm{H}_{2} \mathrm{O}\right)}{5 \times 10^{-7}}\right) \\
& \times\left(\frac{R_{\text {out }}}{R_{\text {int }}}-1\right) \times\left(\frac{4 \times 10^{17} \mathrm{~cm}}{R_{\text {out }}}\right),
\end{aligned}
$$

which gives $\tau_{r} \approx 0.25$ for model $C$. The fraction of o- $\mathrm{H}_{2} \mathrm{O}$ molecules in excited energy levels is $20-30 \%$ at $r \sim 8 \times 10^{15} \mathrm{~cm}$ and increases sharply with diminishing $r$ (Fig. 4ha); therefore, eq. (3) overestimates the opacities for $R_{\text {int }} \lesssim 8 \times 10^{15} \mathrm{~cm}$. Our models yield maximum values of $\tau_{r}=0.30$ for the case of $R_{\text {int }}=4 \times 10^{15} \mathrm{~cm}$. All other ro-vibrational o- $\mathrm{H}_{2} \mathrm{O}$ transitions have excited lower levels and hence lower opacities.

On the other hand, the collisional pumping rate from $1_{01}$ to $1_{10}$ per molecule in the $1_{01}$ level is $\Gamma_{c}=n\left(\mathrm{H}_{2}\right) C_{l u}\left(T_{k}\right)$, where $C_{l u}$ is the collisional rate from the $1_{01}$ to the $1_{10}$ level, and can be written as

$$
\begin{aligned}
\Gamma_{c} & \approx \frac{0.01}{r_{15}^{2}} \times\left(\frac{C_{l u}(300 \mathrm{~K})}{2.3 \times 10^{-10} \mathrm{~cm}^{3} \mathrm{~s}^{-1}}\right) \\
& \times \exp \left\{-120\left(\frac{1}{T_{k}(r)}-\frac{1}{300}\right)\right\} \mathrm{s}^{-1},
\end{aligned}
$$

where the exponential factor is an approximate fit to the variation of $C_{l u}$ with $T_{k}$, valid up to $r \approx 3 \times 10^{16} \mathrm{~cm}$. Equations (2) and (4) show that even at $r_{15}=1$, where $T_{k} \approx 300 \mathrm{~K}$, the radiative-to-collisional pumping rate ratio is $\sim 2$, and strongly increases with increasing $r$ as a consequence of the diminishing $T_{k}$. Figure $4 \mathrm{~b}$ shows the actual values of $\Gamma_{r}$ (dashed line) and $\Gamma_{c}$ (solid line) given by the code in model $A$. At $r_{15}=1, \Gamma_{r}$ is a factor of $\approx 2$ higher than predicted by eq. (2) because dust and gas are mixed and $\langle J\rangle$ is higher than estimated above. The contribution to the excitation of other radiative paths, like $1_{01} \rightarrow \nu_{2} 2_{12} \rightarrow 2_{21} \rightarrow 1_{10}$ and $2_{12} \rightarrow \nu_{2} 1_{01} \rightarrow 1_{10}$, accounts for the relatively low o- $\mathrm{H}_{2} \mathrm{O}$ abundance required to reproduce the observed $557 \mathrm{GHz}$ flux.

As pointed out in section 2, the inclusion of the $\nu_{3}=1$ state has negligible effect on the line fluxes from the ground vibrational state, which can be shown as follows. The strongest line connecting the $1_{01}$ level and the $\nu_{3}=1$ state is the $1_{01} \rightarrow \nu_{3} 2_{02}$ transition at $\lambda=2.63$ $\mu \mathrm{m}$, with a pumping rate per molecule in the $1_{01}$ level given by $\Gamma_{r 3} \propto \lambda^{5} g_{u p} A_{u l} F_{2.6 \mu \mathrm{m}}$; here $g_{u p}$ is the degeneracy of the upper level of the transition, $A_{u l} \approx 33 \mathrm{~s}^{-1}$ is the Einstein coefficient for spontaneous emission, and $F_{2.6 \mu \mathrm{m}} \approx 0.6 \times 10^{-13} \mathrm{~W} \mathrm{~cm}^{-2} \mu \mathrm{m}^{-1}$ is the observed $2.6 \mu \mathrm{m}$ continuum flux. A similar expression applies to the pumping rate in the $1_{01} \rightarrow \nu_{2} 1_{10}$ transition at $6.2 \mu \mathrm{m}\left(A_{u l} \approx 10 \mathrm{~s}^{-1}\right)$, so that the ratio of the pumping rates from the $1_{01}$ level into the $\nu_{2} 1_{10}$ and $\nu_{3} 2_{02}$ levels is found to be $\approx 55$. 
Figure 5 shows the predicted $557 \mathrm{GHz}$ line profiles as observed with $\mathrm{HSO}\left(\mathrm{HPBW}=41^{\prime \prime}\right)$. Results are given for the telescope pointing toward the source center, and shifted $41^{\prime \prime}$ from the source center. In models $A$ and $B$, the contribution to the total emission of the outermost regions $\left(r>4 \times 10^{16} \mathrm{~cm}\right.$, or $\left.16^{\prime \prime}\right)$ to the $557 \mathrm{GHz}$ emission is low, and so the line shapes and relative intensity toward the offset position are typical of an optically thick, spatially unresolved line. Model $C$ shows, on the contrary, a nearly flat line profile with relatively weak emission toward the source center because the source is partially resolved; in this case the intensity toward the offset position is the strongest of the three models. The predicted line flux and shape within the HSO beam is somewhat dependent on the assumed value of $R_{\text {out }}$, but significant loss of line flux in the wings of the beam is expected in model $C$ as long as the source size, $2 \times R_{\text {out }}$, is larger than the beam size at $557 \mathrm{GHz}, 10^{17} \mathrm{~cm}$ (i.e. $\left.R_{\text {out }} / R_{\text {int }}>1.25\right)$.

\subsection{Predictions for other lines in IRC +10216}

The models presented below, showing predictions for $\mathrm{H}_{2} \mathrm{O}$ lines other than the 557 $\mathrm{GHz}$ one, are the same as those already described in the previous sections, with values for the water vapor abundance such that the $\mathrm{o}-\mathrm{H}_{2} \mathrm{O} 557 \mathrm{GHz}$ line flux observed by SWAS is reproduced for all assumed $R_{\text {int }}$ (Fig. 3). Figure 6 shows the energy level diagram of ortho and para- $\mathrm{H}_{2} \mathrm{O}$, and indicates the strongest lines $\left(\Delta K_{-1}= \pm 1, \Delta K_{+1}= \pm 1\right)$ that lie within the HSO-HIFI allowed frequency range. With reference to the energy of the upper level, the lines may be classified into three groups: (i) 8 low-excitation lines, with $E_{u p} \lesssim 200 \mathrm{~K}$; (ii) 3 mid-excitation lines, with $200 \mathrm{~K}<E_{u p} \lesssim 300 \mathrm{~K}$; (iii) 6 high-excitation lines, with $E_{u p}>300$ $\mathrm{K}\left(J_{u p} \geq 4\right)$. Figure 7 plots the predicted line fluxes, as observed with the HSO-HIFI beam, versus the inner radius of the $\mathrm{H}_{2} \mathrm{O}$ shell, and Fig. 8] shows the expected line profiles for models $A, B$, and $C$. Line frequencies and HSO-HIFI beamwidths adopted in these calculations are listed in Table 3 ,

The overall excitation is, as in the case of the $557 \mathrm{GHz}$ line, dominated by the pumping of the $\nu_{2}$ state through absorption of $6 \mu \mathrm{m}$ dust-emitted photons. Nevertheless, absorption of continuum photons in far-infrared pure-rotational transitions also plays an important role in the excitation of some mid- and high-excitation lines. For example, absorption of $45 \mu \mathrm{m}$ and $58 \mu \mathrm{m}$ photons in the 523414 and 422313 lines, contributes significantly to the excitation of the 523514 and 422413 lines, respectively. On the other hand, we find that the effect of collisions is ignorable in all our models. We have run model $A$ by quenching off all collisions among rotational levels, and found that the line fluxes vary by less than $4 \%$. Collisional rates much higher than estimated in our extrapolation would be required for collisions to 
compete with the radiation field. We conclude that the excitation of $\mathrm{H}_{2} \mathrm{O}$ is dominated by the radiation field in all lines and models and our results are dependent on neither the $\mathrm{H}_{2}$ density nor the $T_{k}$ profiles, but rely on the adequate simulation of the radiation field generated by dust.

Line fluxes decrease with increasing $R_{\text {int }}$ because $(i)$ owing to the decrease of the overall $\mathrm{H}_{2} \mathrm{O}$ excitation with increasing distance from the star (Fig. 4a), the emissivity of all lines decreases with increasing $R_{\text {int }}$ more steeply than that of the lowest-lying 110101 transition, and since the SWAS flux of the latter line is kept constant for all $R_{\text {int }}$, all other line fluxes decrease; $(i i)$ for high $R_{\text {int }}$ and low-excitation lines, the emission becomes spatially resolved, and hence line flux is lost beyond the wings of the HSO-HIFI beam. The latter effect becomes quite pronounced for high-frequency lines, and explains why the 212101 line (HPBW=14.. 5 , corresponding to a radius of $\approx 2 \times 10^{16} \mathrm{~cm}$ ) is predicted to be in absorption against the 179.5 $\mu \mathrm{m}$ continuum emission for $R_{\text {int }} \gtrsim 4 \times 10^{16} \mathrm{~cm}$ (Fig. 8).

Lines belonging to different groups trace different ranges of $R_{\text {int }}$. The low-excitation lines will allow us to check whether $\mathrm{H}_{2} \mathrm{O}$ is formed as a result of photodissociation products. If $\mathrm{H}_{2} \mathrm{O}$ is formed in the outermost layers of the envelope, with $R_{\text {int }} \gtrsim 2 \times 10^{16} \mathrm{~cm}$, the 557 $\mathrm{GHz}$ line is expected to be the strongest of all lines. Most lines would be undetectable in such a case, given the expected sensitivity of HSO-HIFI (Table 33).

The observation of the high and mid-excitation lines would check whether $\mathrm{H}_{2} \mathrm{O}$ is formed in the innermost layers of the envelope. The fluxes predicted for the high-excitation lines decrease by one order of magnitude or more for $4.5 \times 10^{14} \mathrm{~cm} \leq R_{\text {int }} \leq 2 \times 10^{15} \mathrm{~cm}$; however, these high-frequency lines are hardly detectable with the expected sensitivity of HSO-HIFI. Also, the predicted fluxes are somewhat uncertain because they depend on the details of the radiation field in the innermost regions of the envelope, which in turn depend on the distribution and emissivity of dust in these regions. Nevertheless, some mid-excitation lines are still sensitive to low values of $R_{\text {int }}$, as, for example, the 312303 and 321312 transitions. These lines, as well as other low-excitation lines, will also probe intermediate values of $R_{\text {int }}$. The fluxes of the 312303 and 312221 lines vary by a factor of $\approx 2.5$ from $R_{\text {int }}=2 \times 10^{15} \mathrm{~cm}$ to $R_{\text {int }}=4 \times 10^{15} \mathrm{~cm}$, and the flux of the 303212 line varies by a factor of $\approx 2$ in the same $R_{\text {int }}$ interval.

The expected line profiles (Fig. 8) display a variety of shapes, from parabolic (opticallythick, spatially-unresolved lines) to triangular (high-excitation lines), double-peaked (spatiallyresolved lines), top-hat (optically-thin, spatially-unresolved lines) and P-Cygni (spatiallyresolved, ground-state lines) profiles. The greater uncertainty concerns the predicted shapes of the high-excitation lines, as they are formed in the acceleration region. 


\subsection{HSO observations of C-rich AGB stars other than IRC+10216}

In this section we explore the prospects for detecting any of the low-lying ground vibrational state $\mathrm{H}_{2} \mathrm{O}$ lines, 110101 at $557 \mathrm{GHz}, 111000$ at $1113 \mathrm{GHz}$, and 212101 at $1670 \mathrm{GHz}$, in C-rich AGB stars other than IRC+10216. Figure 9 shows that, for a source similar to IRC +10216 , the line flux ratios depend for high $R_{\text {int }}$ on the distance to the source, as a consequence of the spatially extended emission and of the different HSO/HIFI beams available for the three lines (Table 3). For $R_{\text {int }} \approx 4 \times 10^{16} \mathrm{~cm}$, the $1670 \mathrm{GHz}$ line will only be spatially unresolved if the source is located at more than $2 \mathrm{kpc}$. We will restrict in the following to models with $R_{\text {int }}=2 \times 10^{15} \mathrm{~cm}$, which predict spatially unresolved emission in all lines at essentially any distance. This value of $R_{\text {int }}$ corresponds to model $B$ in the previous sections, which assumes $\mathrm{H}_{2} \mathrm{O}$ to be formed or released at intermediate radii, and whose results for the low-lying lines considered here are also very similar to those obtained for the lowest $R_{\text {int }}$ (model $A$, see Table 2).

The role of the mid-IR continuum emission in the excitation of $\mathrm{H}_{2} \mathrm{O}$ in IRC +10216 should be also evaluated in other C-rich stars. To this end, we show in Fig. 10a the $6.3 \mu \mathrm{m}$ mid-IR flux, corrected for the distance, versus the mass loss rate for a sample of C-rich AGB stars. Most $\dot{M}$ and $D$ values have been taken from the compilation of Guandalini et al. (2006). Distance estimates were mostly inferred from HIPPARCOS astrometric measurements by Bergeat, Knapik, \& Rutily (2002); the mass loss rates, derived from millimetric molecular observations, were extracted from the literature (e.g., Loup et al. 1993) and subsequently corrected for the updated distances by Bergeat \& Chevallier (2005). Filled symbols in Fig. 10a indicate sources for which the $6.3 \mu \mathrm{m}$ flux has been directly measured from ISO/SWS spectra (Sloan et al. 2003)1. The ISO/SWS spectra has also allowed us to determine the 6.3 -to- $8.8 \mu \mathrm{m}$ flux density ratio as a function of $\dot{M}$. This ratio, ranging from 1.25 for the highest $\dot{M}$ to 3.5 for the lowest $\dot{M}$, has been used to estimate the $6.3 \mu \mathrm{m}$ fluxes, shown with open symbols, for those sources not observed by ISO/SWS but for which the 8.8 $\mu \mathrm{m}$ flux is available (Guandalini et al. 2006; Monnier et al. 1998; López et al. 1993). The $6.3 \mu \mathrm{m}$ emission is responsible for the excitation of $\mathrm{H}_{2} \mathrm{O}$ through the pumping of the $\nu_{2}=1$ state, and is thus a measure of the impact of the mid-IR emission on the excitation of $\mathrm{H}_{2} \mathrm{O}$. The plot shows that IRC +10216 has a high mid-IR intrinsic luminosity relative to other C-rich stars with similar $\dot{M}$. In order to simulate the general trend $L_{6.3 \mu \mathrm{m}}-\dot{M}$ found for the bulk of sources, we have generated dust models that differ in both $\dot{M}$ and the stellar luminosity $\left(L_{*}\right)$. For the aim of simplicity, only $L_{*}$ has been varied as a function of $\dot{M}$, while

\footnotetext{
${ }^{1}$ An Atlas of fully processed ISO/SWS spectra is publicly available at http://isc.astro.cornell.edu/ sloan/library/swsatlas/atlas.html
} 
the rest of dust parameters remain equal to those adopted for IRC+10216 (Table 1). The grey line in Fig. 10a shows the fit found with $L_{*}=3.5 \times 10^{3} \mathrm{~L}_{\odot}$ for $\dot{M}=1.2 \times 10^{-7} \mathrm{M}_{\odot} \mathrm{yr}^{-1}$, and $L_{*}=8 \times 10^{3} \mathrm{~L}_{\odot}$ for $\dot{M}=3 \times 10^{-5} \mathrm{M}_{\odot} \mathrm{yr}^{-1}$.

Figure 10b shows the $6.3 \mu \mathrm{m}$ flux density, corrected for the distance and divided by $\dot{M}$, as a function of $\dot{M}$. At any radial position of a given source, the mid-IR radiation density is expected to be proportional to $F_{6.3 \mu \mathrm{m}} D^{2}$ and the $\mathrm{H}_{2}$ density proportional to $\dot{M}$, so that the quantity in the ordinates of Fig. 10 b is proportional to the radiative-to-collisional pumping rate ratio. If the kinetic temperature profile is not very different from that adopted for IRC +10216 , it is expected that $\mathrm{H}_{2} \mathrm{O}$ in those sources with $F_{6.3 \mu \mathrm{m}} D^{2} / \dot{M}$ similar to or higher than the value associated with IRC+10216 will be radiatively pumped. Figure 10b shows that radiative pumping is expected to dominate over collisional pumping at least for the bulk of sources with $\dot{M} \lesssim 10^{-5} \mathrm{M}_{\odot} \mathrm{yr}^{-1}$.

We have used the above dust models to compute the expected $\mathrm{H}_{2} \mathrm{O}$ emission as a function of $\dot{M}$. The key assumption for the $\mathrm{H}_{2} \mathrm{O}$ models displayed below is that the $\mathrm{H}_{2} \mathrm{O}$ outflow rate (i.e. the total number of $\mathrm{H}_{2} \mathrm{O}$ molecules in the envelope) is independent of $\dot{M}$ and equal to the value found in IRC+10216 (which was required to explain the $557 \mathrm{GHz}$ emission). That assumption is appropriate if the origin of the water vapor is the vaporization of icy bodies, because the amount of comets orbiting a star is expected to be independent of $\dot{M}$, whereas any other formation mechanism involves chemical reactions whose volume rates are proportional to the square of the density. In the cometary hypothesis, the release of $\mathrm{H}_{2} \mathrm{O}$ to the outflow is still a function of the stellar luminosity, which is expected to increase with $\dot{M}$, but also depends on other parameters such as the evolutionary stage of the star after the onset of the TP-AGB phase (Ford \& Neufeld 2001), and thus a correlation between the $\mathrm{H}_{2} \mathrm{O}$ outflow rate and $\dot{M}$ is uncertain but probably less pronounced than for any other formation mechanism.

Another important model assumption is the parametrization of the photodissociation radius for $\mathrm{H}_{2} \mathrm{O}$ molecules, $R_{\text {out }}$, as a function of the mass loss rate. Following Netzer \& Knapp (1987), we have assumed that $R_{\text {out }}$ scales as $\dot{M}^{0.7}$, but normalized the values of $R_{\text {out }}$ in such a way that $R_{\text {out }}=4 \times 10^{17} \mathrm{~cm}$ for $\dot{M}=3 \times 10^{-5} \mathrm{M}_{\odot} \mathrm{yr}^{-1}$ (Willacy 2004, AC06). As a result of this different normalization, our values for $R_{\text {out }}$ are one order of magnitude larger than the radius of the $\mathrm{OH}$ shell calculated by Netzer \& Knapp (1987). For high $\dot{M}$, our resulting values for $R_{\text {out }}$ are much larger than the assumed $R_{\text {int }}=2.1 \times 10^{15} \mathrm{~cm}$, and results are not sensitive to the adopted value for $R_{\text {out }}$. However, for $\dot{M} \lesssim 10^{-6} \mathrm{M}_{\odot} \mathrm{yr}^{-1}$, the finite size for the $\mathrm{H}_{2} \mathrm{O}$ shell (e.g. $R_{\text {out }} / R_{\text {int }} \lesssim 10$ ) starts to lower the expected line fluxes, in particular that of the $557 \mathrm{GHz}$ line. Since Netzer \& Knapp (1987) obtain values for $R_{\text {out }}$ that are still much lower than ours, one should consider with caution the $\mathrm{H}_{2} \mathrm{O}$ fluxes predicted for $\dot{M} \lesssim 10^{-6}$ 
$\mathrm{M}_{\odot} \mathrm{yr}^{-1}$. Nevertheless, we note that the photodissociation rate in the unshielded interstellar radiation field $\left(5.9 \times 10^{-10} \mathrm{~s}^{-1}\right)$ yields, for a $15 \mathrm{~km} \mathrm{~s}^{-1}$ flow, a lengthscale of $\sim 2 \times 10^{15} \mathrm{~cm}$. For $R_{\text {int }}=2 \times 10^{15} \mathrm{~cm}$, this implies a minimum value for $R_{\text {out }}$ of $\sim 4 \times 10^{15} \mathrm{~cm}$ for any $\dot{M}$ however small, unless the radiation field is stronger than the average value. For the lowest $\dot{M}=1.2 \times 10^{-7} \mathrm{M}_{\odot} \mathrm{yr}^{-1}$ considered here, the adopted $R_{\text {out }}$ is $8.4 \times 10^{15} \mathrm{~cm}$.

Figure 11 shows the expected fluxes of the three ground-state lines of $\mathrm{H}_{2} \mathrm{O}$ as a function of a) $\dot{M}$, and b) the $6.3 \mu \mathrm{m}$ continuum flux; results are given for a source at $D=0.5 \mathrm{kpc}$. We find that the excitation is dominated by absorption of mid-IR photons; only for the highest $\dot{M}$ does collisional excitation account for at most $20 \%$ of the predicted flux in the groundstate lines. Therefore, a tight correlation between line fluxes and the $6.3 \mu \mathrm{m}$ continuum flux density is found in Fig. 11b, where fits of the form $F \propto F_{6.3 \mu m}^{b}$ are displayed. The slope $b$ is $\approx 1$ for the $1670 \mathrm{GHz}$ line, but somewhat lower for the 557 and $1113 \mathrm{GHz}$ lines $(0.87$ and 0.94 , respectively) due to slight opacity effects in the vibrational lines responsible for the radiative pumping. The slopes would approach to unity for a microturbulent velocity higher than the assumed value of $0.65 \mathrm{~km} \mathrm{~s}^{-1}$. For low $\dot{M}$, the data points are slightly below the fitted lines as a result of the $\mathrm{H}_{2} \mathrm{O}$ photodissociation effects mentioned above; stronger departures from the fitted lines are expected for lower values of $R_{\text {out }}$.

According to the current estimates of the HSO-HIFI sensitivities (Table 3), the $557 \mathrm{GHz}$ line will be detected in 1.5 hours at more than $5 \sigma$ level if the line flux is higher than $1.3 \times 10^{-22}$ $\mathrm{W} \mathrm{cm} \mathrm{cm}^{-2}$ (after coadding the two polarization modes). From Fig. 11b, this sensitivity limit translates into a $6.3 \mu \mathrm{m}$ flux density of $1.7 \times 10^{-15} \mathrm{~W} \mathrm{~cm}^{-2} \mu \mathrm{m}^{-1}$ at $D=0.5 \mathrm{kpc}$, which can be in turn adapted to any other distance provided that both the line and continuum fluxes vary as $D^{-2}$. Figure 11k shows the $6.3 \mu \mathrm{m}$ fluxes for the sample of C-rich AGB stars, with the solid line indicating the $6.3 \mu \mathrm{m}$ flux density required to detect the $557 \mathrm{GHz}$ line according to the quoted sensitivity limit. All sources above the solid line would be detected with 1.5 hours of observing time in the $557 \mathrm{GHz}$ line if the $\mathrm{H}_{2} \mathrm{O}$ outflow rate were equal or higher than the value found in IRC+10216, and if the photodissociation radius of the $\mathrm{H}_{2} \mathrm{O}$ molecules remains sufficiently large relative to $R_{\text {int }}$. The sensitivity limits for the 1113 and $1670 \mathrm{GHz}$ lines are also displayed, and show that the detection of these lines require significantly higher $6.3 \mu \mathrm{m}$ continuum fluxes. We conclude that the $557 \mathrm{GHz}$ line provides the most sensitive means of searching for $\mathrm{H}_{2} \mathrm{O}$ in C-rich AGB stars other than IRC+10216 with HSO. 


\section{Discussion}

\section{1. $\quad$ Model uncertainties}

Our models for IRC+10216 predict that, whatever the region where $\mathrm{H}_{2} \mathrm{O}$ is formed or released to the outflow, $\mathrm{H}_{2} \mathrm{O}$ is radiatively excited, which implies that model results are independent of the gas temperature and density profiles. This result relies on the extrapolation to higher $T_{k}$ of the collisional rates given by Phillips et al. (1996), which will require further confirmation from new estimates of $\mathrm{H}_{2} \mathrm{O}-\mathrm{H}_{2}$ collisional rates at higher temperatures. Nevertheless, we find that collisional rates would have to be about one order of magnitude higher than estimated to compete efficiently with the radiative rates in the innermost regions of the envelope, which seems somewhat implausible.

A relatively uncertain parameter in our models is the assumed distance to IRC +10216 , $D=170 \mathrm{pc}$. It has been proposed that the distance may be substantially smaller, $D=$ $100-150$ pc (Zuckerman, Dyck, \& Claussen 1986; Groenewegen et al. 1992). At 170 pc, the inferred stellar luminosity is $L_{*} \approx 2 \times 10^{4} \mathrm{~L}_{\odot}$, which results in $L_{*} \approx 10^{4} \mathrm{~L}_{\odot}$ at $D=120 \mathrm{pc}$, a value more similar to the inferred luminosities of other C-rich AGB stars with similar $\dot{M}$ (Schöier, Olofsson, \& Lundgren 2006). If $D=120$ pc, both $\dot{M}$ and the radiation density at $6 \mu \mathrm{m}$ are a factor of 2 lower than assumed, so that the radiative-to-collisional pumping rate ratio still remains unchanged. Since the closer proximity and weaker $6 \mu \mathrm{m}$ radiation density have opposite effects on the $\mathrm{H}_{2} \mathrm{O}$ outflow rate required to match the SWAS $557 \mathrm{GHz}$ flux, the latter will decrease by less than a factor 2 , and thus the expected $\mathrm{H}_{2} \mathrm{O}$ abundance will be a factor of $<2$ higher than in Table 2. Therefore, we conservatively estimate an o- $\mathrm{H}_{2} \mathrm{O}$ abundance in the range $(0.5-1) \times 10^{-7}$ for $R_{\text {int }}=2 \times 10^{15} \mathrm{~cm}$. Concerning the line ratios to be observed by HSO, we expect values similar to those obtained at $D=170 \mathrm{pc}$ if the line emission remains unresolved, i.e. for low values of $R_{\text {int }}$. For high values of $R_{\text {int }}$, beam effects will be more important at $D=120 \mathrm{pc}$, diminishing the low-excitation line fluxes relative to the $557 \mathrm{GHz}$ line.

A potentially more important source of uncertainty is the assumption of spherical symmetry in our models. Both the molecular and dust emission from IRC+10216 show evidence for departures from a smooth distribution in a spherically symmetric outflow; incomplete, discrete shells or arcs and clumpy structures are instead observed on a wide range of distances to the star (e.g. Lucas \& Guélin 1999; Mauron \& Huggins 1999; Fong. Meixner, \& Shah 2003; Leão et al. 2006). Even more important, the OH line shapes observed in IRC+10216 strongly suggest an asymmetric distribution of $\mathrm{OH}$ in the outer regions of the envelope (Ford et al. 2003), thus also suggesting an asymmetric distribution of the parent $\mathrm{H}_{2} \mathrm{O}$ molecule. These asymmetries may alter to some extent the $\mathrm{H}_{2} \mathrm{O}$ line flux ratios calculated with the use of 
our spherically symmetric approach. While in spherical symmetry the emission from any line is isotropic, in filamentary structures or slabs of velocity-coherent gas the optically thick lines radiate preferentially in the direction perpendicular to the two faces of the sheet (Elitzur, Hollenbach, \& McKee 1989), whereas the emission from thinner lines will approach a more isotropic behavior. Future HSO observations will show the importance of the observed morphological complexity on the line fluxes by showing whether the different $\mathrm{H}_{2} \mathrm{O}$ line flux ratios are consistent with a single value of $R_{\text {int }}$, or indicate a range of values.

Finally, the models assume a water shell with uniform $\mathrm{H}_{2} \mathrm{O}$ abundance and sharp inner and outer radii; however, a finite abundance gradient obviously takes place at both the $\mathrm{H}_{2} \mathrm{O}$ formation and dissociation regions, and variations of the $\mathrm{H}_{2} \mathrm{O}$ abundance across the shell are also possible. These effects may also alter to some extent the expected line flux ratios. Uncertainties in the outer radius of the $\mathrm{H}_{2} \mathrm{O}$ shell may also affect the expected fluxes from stars with low mass loss rates.

\subsection{Water formation at inner or intermediate radii}

Both the cometary and Fischer-Tropsch catalysis hypothesis predict $\mathrm{H}_{2} \mathrm{O}$ to be released or formed at intermediate radii of a few $\times 10^{15} \mathrm{~cm}$, and although no specific mechanism has been proposed for $\mathrm{H}_{2} \mathrm{O}$ formation at the innermost regions $\left(R_{\text {int }}<10^{15} \mathrm{~cm}\right)$, this possibility cannot be rejected. The models shown in section 3.2 will permit us to discriminate, from HSO observations of IRC +10216 , if there are significant amounts of $\mathrm{H}_{2} \mathrm{O}$ in the innermost regions through the observation of mid-excitation $\mathrm{H}_{2} \mathrm{O}$ transitions. More difficult will be a priori to discriminate between the cometary and Fischer-Tropsch catalysis propositions. Both predict similar values for $R_{\text {int }}$; nevertheless, if $R_{\text {int }}$ were found to be significantly higher than $2 \times 10^{15}$ $\mathrm{cm}$, the release of $\mathrm{H}_{2} \mathrm{O}$ from comets could be favoured, unless some additional mechanism were found to shift $R_{\text {int }}$, within the Fischer-Tropsch catalysis framework (Willacy 2004), outwards in the envelope. The search for water emission at $557 \mathrm{GHz}$ in C-rich AGB stars other than IRC +10216 will also favour one of the two hypotheses: since IRC +10216 is at the high end of mass loss rates from C-rich stars, and the efficiency of Fischer-Tropsch catalysis to form $\mathrm{H}_{2} \mathrm{O}$ molecules is expected to decrease with diminishing $\dot{M}$, one would expect in such a case a rate of detection significantly lower than that quantified in section 3.3 for the cometary hypothesis, and one would expect a pronounced decline of the $\mathrm{H}_{2} \mathrm{O}$ abundance with diminishing $\dot{M}$.

The ISO/LWS spectrum of IRC+10216 (Cernicharo et al. 1996) shows an emission feature at $179.5 \mu \mathrm{m}$, coincident with the wavelength of the $212101 \mathrm{o}^{-} \mathrm{H}_{2} \mathrm{O}$ transition, with a flux of $\approx 8 \times 10^{-20} \mathrm{~W} \mathrm{~cm}^{-2}$. This flux is very similar to that computed for the quoted line 
in model $B\left(R_{\text {int }}=2.1 \times 10^{15} \mathrm{~cm}\right)$. However, the far-infrared spectrum of IRC +10216 shows vibrationally excited rotational emission of $\mathrm{HCN}$, and the combined emission of the $\nu_{1}=1$ $J=19 \rightarrow 18$ and $\nu_{3}=1 J=19 \rightarrow 18 \mathrm{HCN}$ lines, both emitting at $179.5 \mu \mathrm{m}$, is expected to be also comparable to the measured line flux at $179.5 \mu \mathrm{m}($ Cernicharo et al. 1996). Given the uncertainties inherent to the HCN model in Cernicharo et al. (1996), where the excited vibrational states are assumed to be thermalized, and given the high density of spectral lines in the IRC+10216 far-infrared spectrum, which makes it difficult to establish the contribution from the $\nu_{1}=1$ and $\nu_{3}=1$ rotational lines to the spectrum, the relative contribution from $\mathrm{HCN}$ and $\mathrm{H}_{2} \mathrm{O}$ to the observed spectral feature is quite uncertain. On the other hand, the expected flux of the 303212 line at $174.6 \mu \mathrm{m}$ in model $B$ is $\approx 4 \times 10^{-20} \mathrm{~W} \mathrm{~cm}^{-2}$, below the $3-\sigma$ upper limit derived for that line from the ISO/LWS spectrum. These considerations suggest weakly that $\mathrm{H}_{2} \mathrm{O}$ in $\mathrm{IRC}+10216$ is formed or released at radial distances $R_{\text {int }} \gtrsim 2 \times 10^{15} \mathrm{~cm}$.

Our models show that the required $\mathrm{H}_{2} \mathrm{O}$ abundance for $R_{\text {int }}$ in the range $(2-5) \times 10^{15}$ $\mathrm{cm}$ is $(0.5-1) \times 10^{-7}$ relative to $\mathrm{H}_{2}$, which makes the requirements previously demanded for the cometary and Fischer-Tropsch catalysis hypothesis to work in IRC+10216 less restrictive (Ford \& Neufeld 2001; Willacy 2004). The water outflow rate is $(0.5-1) \times 10^{-5} \mathrm{M}_{\oplus} \mathrm{yr}^{-1}$, which yields, within the framework of vaporization of icy bodies, a required total initial ice mass of $(0.5-10) \mathrm{M}_{\oplus}$ for $\dot{M}\left(\mathrm{H}_{2} \mathrm{O}\right) / M_{0}$ (ice) in the range $10^{-5}-10^{-6} \mathrm{yr}^{-1}$ (Ford \& Neufeld 2001). On the other hand, the Fischer-Tropsch catalysis on metallic grains will require a density of iron grains relative to total $\mathrm{H}$ nuclei of $n_{g}(\mathrm{Fe}) / n_{\mathrm{H}}=(0.5-1) \times 10^{-13}$ to explain the observed $\mathrm{H}_{2} \mathrm{O} 557 \mathrm{GHz}$ emission (Willacy 2004).

\subsection{Water formation in the outermost layers}

Multitransition $\mathrm{HSO}$ observations of $\mathrm{H}_{2} \mathrm{O}$ in $\mathrm{IRC}+10216$ will easily establish whether or not water is formed in the external layers of the envelope; the predicted fluxes in Fig. 7 and the HSO-HIFI sensitivities in Table 3 indicate that only the $110101 \mathrm{o}^{-} \mathrm{H}_{2} \mathrm{O}$ line, and possibly the 111000 and $202111 \mathrm{p}-\mathrm{H}_{2} \mathrm{O}$ lines, are detectable in one hour of observing time for $R_{\text {int }} \gtrsim 4 \times 10^{16} \mathrm{~cm}$.

For high $R_{\text {int }}$, the expected flux in the $557 \mathrm{GHz}$ line can be obtained analytically. Since

essentially all o- $\mathrm{H}_{2} \mathrm{O}$ molecules are in the ground $1_{01}$ level (Fig. 4a), the o- $\mathrm{H}_{2} \mathrm{O} 110101$ line flux is derived from the radiative pumping rate given in eq. (2) after multiplying $\Gamma_{r}$ by 1.35 to account for the two radiative pumping routes that are relevant at high distances from the star (Fig. 4b):

$$
F\left(1_{10} \rightarrow 1_{01}\right)=1.4 \times 10^{-21} \times\left(\frac{4 \times 10^{17} \mathrm{~cm}}{R_{\text {out }}}\right)
$$




$$
\times\left(\frac{R_{\text {out }}}{R_{\text {int }}}-1\right) \times\left(\frac{X\left(\mathrm{o}-\mathrm{H}_{2} \mathrm{O}\right)}{5 \times 10^{-7}}\right) \frac{\mathrm{W}}{\mathrm{cm}^{2}},
$$

where $X$ is the abundance relative to $\mathrm{H}_{2}$. Equation (5) is independent of the assumed distance $D$ to the star because, in order to match the observed mid-IR continuum, $\Gamma_{r} \propto D^{2}$ (eq. 11); it assumes a water shell with sharp edges at $R_{\text {int }}$ and $R_{\text {out }}$ and constant $\mathrm{H}_{2} \mathrm{O}$ abundance; it ignores slight opacity effects in the ro-vibrational lines (section 3.1) as well as beam effects (both slightly raise the required abundance), and overestimates by less than $20 \%$ the 557 $\mathrm{GHz}$ line flux obtained in model $C$. In the limit $R_{\text {out }} \rightarrow \infty$, and using $F\left(1_{10} \rightarrow 1_{01}\right)=10^{-20}$ $\mathrm{W} \mathrm{cm}^{-2}$ (Melnick et al. 2001), eq. (5) gives

$$
\chi\left(\mathrm{H}_{2} \mathrm{O}\right) \gtrsim 2.4 \times 10^{-7} \times\left(\frac{R_{\text {int }}}{4 \times 10^{16} \mathrm{~cm}}\right),
$$

where $\chi\left(\mathrm{H}_{2} \mathrm{O}\right)$ is here the $\mathrm{H}_{2} \mathrm{O}$ (ortho+para) abundance relative to $\mathrm{H}$ nuclei. Equation 6 gives the sharp-inner edge, lower limit for the $\mathrm{H}_{2} \mathrm{O}$ abundance required to account for the observed $557 \mathrm{GHz}$ line flux, as a function of $R_{\text {int }}$. AC06 have reported an abundance of $\chi\left(\mathrm{H}_{2} \mathrm{O}\right) \sim 10^{-7}$ to account for the observed $557 \mathrm{GHz}$ line flux in IRC +10216 . However, the quoted abundance would imply an inner radius of $R_{\text {int }} \lesssim 2 \times 10^{16} \mathrm{~cm}$, but the $\mathrm{H}_{2} \mathrm{O}$ abundance shown by AC06 (their Fig. 7) decreases sharply at $r<4 \times 10^{16} \mathrm{~cm}$. Based on detailed modelling, we indicate that the $\chi\left(\mathrm{H}_{2} \mathrm{O}\right)$ profile given by AC06 has to be shifted up by a factor of $\approx 2.5$ to account more accurately for the $557 \mathrm{GHz}$ line flux measured by SWAS.

In the model proposed by AC06, atomic oxygen is produced in a shell by the photodissociation of $\mathrm{CO}$ (and particularly the ${ }^{13} \mathrm{CO}$ isotopologue, which shields itself far less effectively than ${ }^{12}$ CO. $)$ Since the temperature is low $(\sim 10 \mathrm{~K})$ within the shell where the atomic oxygen abundance is significant, the neutral-neutral reaction sequence

$$
\begin{gathered}
\mathrm{O}+\mathrm{H}_{2} \rightarrow \mathrm{OH}+\mathrm{H} \\
\mathrm{OH}+\mathrm{H}_{2} \rightarrow \mathrm{H}_{2} \mathrm{O}+\mathrm{H}
\end{gathered}
$$

is very slow -and therefore is negligible as a source of $\mathrm{H}_{2} \mathrm{O}$ - the first reaction being endothermic and the second -although exothermic-- possessing a substantial activation energy barrier.

AC06 therefore proposed the radiative association reaction

$$
\mathrm{O}+\mathrm{H}_{2} \rightarrow \mathrm{H}_{2} \mathrm{O}+\gamma
$$

as an alternative source of $\mathrm{H}_{2} \mathrm{O}$. To match the SWAS- and ODIN-observed water line fluxes, AC06 had to posit that this reaction is relatively rapid at low temperature, with a rate 
coefficient $k_{r a} \sim 10^{-15} \mathrm{~cm}^{3} \mathrm{~s}^{-1}$. According to our estimation above, $k_{r a}$ has to be a factor $\approx 2.5$ higher than this value to account for the measured $110101 \mathrm{H}_{2} \mathrm{O}$ flux. Nevertheless, and even with the use of the $k_{r a}$ estimation given by AC06, we find that this large reaction rate coefficient for the radiative association of $\mathrm{O}$ and $\mathrm{H}_{2}$ is inconsistent with observations of $\mathrm{H}_{2} \mathrm{O}$ and $\mathrm{OH}$ in at least one translucent molecular cloud, for which sufficient data exist to disipate any significant ambiguity: observations of the cloud along the sight-line to HD 154368 -carried out by Spaans et al. (1998) with the use of the Goddard High Resolution Spectrograph (GHRS) on the Hubble Space Telescope (HST) - yield a $3 \sigma$ upper limit on the water abundance that lies almost two orders of magnitude below the value that would obtain were $k_{r a}$ as large as $10^{-15} \mathrm{~cm}^{3} \mathrm{~s}^{-1}$. The factor of discrepancy would rise above 150 for $k_{r a} \approx 2.5 \times 10^{-15} \mathrm{~cm}^{3} \mathrm{~s}^{-1}$.

The best-fit model for the HD 154368 cloud obtained by Spaans et al. (1998) posits a plane parallel cloud of total visual extinction $A_{V}=2.65 \mathrm{mag}$ in which the density of $\mathrm{H}$ nuclei is $n_{\mathrm{H}}=325 \mathrm{~cm}^{-3}$ (this is probably a lower limit at the cloud center, as the density inferred from the $\mathrm{CO} J=1 \rightarrow 0 / 3 \rightarrow 2$ ratio is $\sim 10^{3} \mathrm{~cm}^{-3}$; see van Dishoeck et al. 1991) and the external ultraviolet radiation field is 3 times mean interstellar value given by Draine (1978): $I_{U V}=3$. Under these conditions, the destruction of $\mathrm{H}_{2} \mathrm{O}$ is dominated by photodissociation at a rate $\zeta=5.9 \times 10^{-10} \mathrm{~s}^{-1} \times I_{U V} \times\left(\exp \left\{-1.7 A_{V 1}\right\}+\exp \left\{-1.7 A_{V 2}\right\}\right) / 2$ (Le Teuff, Millar, \& Markwick 2000)2, where $A_{V 1}$ is the visual extinction to one cloud surface and $A_{V 2}=2.65-A_{V 1}$ is the extinction to the other. At the cloud center, the water photodissociation rate is $1.9 \times 10^{-10} \mathrm{~s}^{-1}$.

For a radiative association rate of $k_{r a}$, the ratio of water vapor to atomic oxygen is therefore given by the expression

$$
n\left(\mathrm{H}_{2} \mathrm{O}\right) / n(\mathrm{O})=k_{r a} n_{\mathrm{H}} f_{\mathrm{H}_{2}} / \zeta
$$

where $f_{\mathrm{H}_{2}} \equiv n\left(\mathrm{H}_{2}\right) / n_{\mathrm{H}}$. At the cloud center, the cloud is almost fully molecular, with $f_{\mathrm{H}_{2}} \sim 0.5$, and the above equation yields $n\left(\mathrm{H}_{2} \mathrm{O}\right) / n(\mathrm{O})=8.7 \times 10^{-4}\left(k_{r a} / 10^{-15} \mathrm{~cm}^{3} \mathrm{~s}^{-1}\right)$. In Fig. 12, we show the predicted $n\left(\mathrm{H}_{2}\right) / n_{\mathrm{H}}$ ratio for the best-fit Spaans et al. (1998) model, together with the $n\left(\mathrm{H}_{2} \mathrm{O}\right) / n(\mathrm{O})$ ratio that would result if $k_{r a}$ were $10^{-15} \mathrm{~cm}^{3} \mathrm{~s}^{-1}$. The model predicts hydrogen to be predominantly in molecular form, in agreement with results by Snow et al. (1996). Averaging the $n\left(\mathrm{H}_{2} \mathrm{O}\right) / n(\mathrm{O})$ ratio over the entire sight-line, we obtain a column density ratio $N\left(\mathrm{H}_{2} \mathrm{O}\right) / N(\mathrm{O})=5.3 \times 10^{-4}\left(k_{r a} / 10^{-15} \mathrm{~cm}^{3} \mathrm{~s}^{-1}\right)$.

\footnotetext{
${ }^{2}$ We note that the factor of 1.7 in the exponential, widely used in the literature, yields $\mathrm{H}_{2} \mathrm{O}$ photodissociation rates at the midplane of the plane-parallel cloud that are higher than the values reported by Roberge et al. (1991) by factors of 2.2 and 10 for $A_{V}^{\text {tot }}=1 \mathrm{mag}$ and $A_{V}^{\text {tot }}=10 \mathrm{mag}$, respectively; therefore, our estimation for the $N\left(\mathrm{H}_{2} \mathrm{O}\right) / N(\mathrm{O})$ ratio predicted by the radiative association of $\mathrm{O}$ and $\mathrm{H}_{2}$ is probably a conservative lower limit.
} 
The atomic oxygen column density along the HD 154368 sight-line is $N(\mathrm{O})=1.2 \times$ $10^{18} \mathrm{~cm}^{-2}$ (Snow et al. 1996, from absorption line observations of the $\left.1355 \AA \mathrm{OI}\right]$ line). Based on a search for the $C^{1} B_{1}-X^{1} A_{1}$ band of water vapor near $1240 \AA$, Spaans et al. (1998) obtained $3 \sigma$ upper limit on the water column density of $9 \times 10^{12} \mathrm{~cm}^{-2}$, corresponding to $N\left(\mathrm{H}_{2} \mathrm{O}\right) / N(\mathrm{O})=7.5 \times 10^{-6}$. This $3 \sigma$ upper limit lies a factor 70 below the value that we would obtain were $k_{r a}$ equal to $10^{-15} \mathrm{~cm}^{3} \mathrm{~s}^{-1}$ as AC06 suggested, and places a $3 \sigma$ upper limit of $1.4 \times 10^{-17} \mathrm{~cm}^{3} \mathrm{~s}^{-1}$ on $k_{r a}$.

AC06 suggested that the freeze out of oxygen onto grain mantles could diminish the water vapor abundance in molecular clouds, as proposed by Bergin et al. (2000) to explain the low $\mathrm{H}_{2} \mathrm{O}$ abundances measured by SWAS in dense clouds. Ice absorption line observations of diffuse/translucent sight-lines, however, indicate that water ice is generally present only in clouds of $A_{V} \geq 3$ (Whittet et al. 2001). Furthermore, in the specific case of HD 154368 under present consideration, the atomic oxygen is known from direct measurement to be $1.2 \times 10^{18} \mathrm{~cm}^{-2}$. The column density of $\mathrm{H}$ nuclei along this sight-line, $N_{\mathrm{H}}=N(\mathrm{H})+2 N\left(\mathrm{H}_{2}\right)$, has been measured to be $4.2 \times 10^{21} \mathrm{~cm}^{-2}$ (Snow et al. 1996), so the mean line-of-sight oxygen abundance is $n(\mathrm{O}) / n_{\mathrm{H}} \sim 3 \times 10^{-4}$, a value that is entirely consistent with the abundances measured along diffuse sight-lines (Meyer et al. 1998) and inconsistent with a significant depletion of oxygen onto ice mantles.

In summary, the upper limit on the water vapor abundance observed towards HD 154368 definitively rules out a rate coefficient for the radiative association reaction $\mathrm{O}+\mathrm{H}_{2} \rightarrow \mathrm{H}_{2} \mathrm{O}+\gamma$ that is large enough to explain -in the context of the AC06 model- the $\mathrm{H}_{2} \mathrm{O} 110101$ line strength measured by SWAS toward IRC+10216. If HSO observations would indicate that $\mathrm{H}_{2} \mathrm{O}$ is formed in the external layers of $\mathrm{IRC}+10216$, an explanation other than the radiative association proposed by AC06 would be required to avoid incompatibilities with observations toward HD 154368.

\section{Summary}

Our radiative transfer models for $\mathrm{H}_{2} \mathrm{O}$ in IRC+10216 and other C-rich AGB stars reveal: 1) $\mathrm{H}_{2} \mathrm{O}$ in the envelope of IRC +10216 is primarily excited through absorption of photons in the $\nu_{2}$ band at $6 \mu \mathrm{m}$ and subsequent decay to the ground vibrational state.

2) The o- $\mathrm{H}_{2} \mathrm{O}$ abundance relative to $\mathrm{H}_{2}$ required to account for the $557 \mathrm{GHz} 110101 \mathrm{o}-\mathrm{H}_{2} \mathrm{O}$ line observed in $\mathrm{IRC}+10216$ is in the range $(0.5-5) \times 10^{-7}$, depending on the inner radius of the $\mathrm{H}_{2} \mathrm{O}$ shell.

3) Multitransition $\mathrm{H}_{2} \mathrm{O}$ observations with the Herschel Space Observatory will allow us to establish the spatial distribution of $\mathrm{H}_{2} \mathrm{O}$ molecules in $\mathrm{IRC}+10216$, in particular the inner 
radius of the $\mathrm{H}_{2} \mathrm{O}$ shell, thus discriminating among the different hypothesis proposed for the origin (formation or release) of $\mathrm{H}_{2} \mathrm{O}$.

4) A number of other C-rich AGB stars with relatively strong $6 \mu \mathrm{m}$ continuum flux are expected to be detectable in the $110101 \mathrm{o}-\mathrm{H}_{2} \mathrm{O}$ line with $\mathrm{HSO}$, if the $\mathrm{H}_{2} \mathrm{O}$ outflow rate in those sources is similar to that found in IRC +10216 .

5) The relatively low $\mathrm{H}_{2} \mathrm{O}$ abundance required to explain the $110101 \mathrm{o}-\mathrm{H}_{2} \mathrm{O}$ emission in IRC +10216 makes less restrictive the requirements previously reported for the vaporization of icy bodies or Fischer-Tropsch catalysis on the surfaces of metallic grains to work. If $\mathrm{H}_{2} \mathrm{O}$ were formed in the external layers of IRC+10216, we find that an explanation for the $\mathrm{H}_{2} \mathrm{O}$ formation other than the previously reported radiative association $\mathrm{O}+\mathrm{H}_{2} \rightarrow \mathrm{H}_{2} \mathrm{O}+\gamma$ is required to avoid conflict with observations of the translucent cloud towards HD 154368.

D.A.N. gratefully acknowledges the support of grant NAG 5 - 13114 from NASA's Long Term Space Astrophysics research program. This research has made use of NASA's Astrophysics Data System.

\section{REFERENCES}

Agúndez, M., \& Cernicharo, J. 2006, ApJ, 650, 374 (AC06)

Bagnulo, S., Doyle, J.G., \& Griffin, L.P. 1995, A\&A, 301, 501

Bergeat, J., Knapik, A., \& Rutily B. 2002, A\&A, 390, 967

Bergeat, J., \& Chevallier, L. 2005, A\&A, 429, 235

Bergin, E.A., Melnick, G.J., Stauffer, J.R., et al. 2000, ApJ, 539, L129

Cernicharo, J., Barlow, M.J., González-Alfonso, E., et al. 1996, A\&A, 315, L201

Dinh-V-Trung, \& Nguyen-Q-Rieu 2000, A\&A, 361, 601

Draine, B.T. 1978, ApJS, 36, 595

Elitzur, M., Hollenbach, D.J., McKee, C.F. 1989, ApJ, 346, 983

Fong, D., Meixner, M., \& Shah, R.Y. 2003, ApJ, 582, L39

Ford, K.E.S., Neufeld, D.A. 2001, ApJ, 557, L113

Ford, K.E.S., Neufeld, D.A., Goldsmith, P.F., \& Melnick, G.J. 2003, ApJ, 589, 430 
Ford, K.E.S., Neufeld, D.A., Schilke, P., \& Melnick, G.J. 2004, ApJ, 614, 990

Glassgold, A.E. 1996, ARAA, 34, 241

González-Alfonso, E., \& Cernicharo, J. 1997, A\&A, 322, 938

González-Alfonso, E., \& Cernicharo, J. 1999, ESA SP-427, "The Universe as seen by ISO”, 325

González-Alfonso, E., \& Cernicharo, J. 1999, ApJ, 525, 845

Green, S., Maluendes, S., \& McLean, A.D. 1993, ApJS, 85, 181

Groenewegen, M.A.T., de Jong, T., van der Bliek, N.S., Slijkhuis, \& S., Willems, F.J. 1992, A\&A, 253, 150

Guandalini, R., Busso, M., Ciprini, S., Silvestro, G., \& Persi, P. 2006, A\&A, 445, 1069

Hasegawa, T.I., Kwok, S., Koning, N., et al. 2006, ApJ, 637, 791

Kwan, J., \& Linke, R.A. 1982, ApJ, 254, 587

Leão, I.C., de Laverny, P., Mékarnia, D., de Medeiros, J.R., \& Vandame, B. 2006, A\&A, 455,187

Le Teuff, Y.H., Millar, T.J., \& Markwick, A.J. 2000, A\&AS, 146, 157

López, B., Perrier, C., Mekarnia, D., Lefevre, J., \& Gay, J. 1993, A\&A, 270, 462

Loup, C., Forveille, T., Omont, A., \& Paul, J.F. 1993, A\&AS, 99, 291

Lucas, R., Guélin, M. 1999, in IAU Symposium 191, Ed. by Le Bertre, T., Lebre, A., \& Waelkens, C. IAU \& Astr. Soc. of the Pacific, p. 305

Mauron, N., \& Huggins, P.J. 1999, A\&A, 349, 203

Melnick, G.J., Neufeld, D.A., Ford, K.E.S., Hollenbach, D.J., Ashby, M.L.N. 2001, Nature, 412, 160

Meyer, D.M., Jura, M., \& Cardelli, J.A. 1998, ApJ, 493, 222

Monnier, J.D., Geballe, T.R., \& Danchi, W.C. 1998, ApJ, 502, 833

Netzer, N., \& Knapp, G.R. 1987, ApJ, 323, 734

Phillips, T.R., Maluendes, S., \& Green, S. 1996, ApJS, 107, 467 
Roberge, W.G., Jones, D., Lepp, S., \& Dalgarno, A. 1991, ApJS, 77, 287

Rothman, L.S., et al. 2005, Journal of Quantitative Spectroscopy \& Radiative Transfer, 96, 139

Schöier, F.L., Olofsson, H., \& Lundgren, A.A. 2006, A\&A, 454, 247

Schönberg, K., \& Kempe, K. 1986, A\&A, 163, 151

Sloan, G.C., Kraemer, K.E., Price, S.D., \& Shipman, R.F. 2003, ApJS, 147, 379

Snow, T.P., Black, J.H., van Dishoeck, E.F., Burks, G., Crutcher, R.M., Lutz, B.L., Hanson, M.M., \& Shuping, R.Y. 1996, ApJ, 465, 245

Spaans, M., Neufeld, D., Lepp, S., Melnick, G.J., \& Stauffer, J. 1998, ApJ, 503, 780

Tenenbaum, E.D., Apponi, A.J., Ziurys, L.M., Agúndez, M., Cernicharo, J., Pardo, J.R., \& Guélin, M. 2006, ApJ, 649, L17

van Dishoeck, E.F., Phillips, T.G., Black, J.H., \& Gredel, R. 1991, ApJ, 366, 141

Whittet, D.C.B., Gerakines, P.A., Hough, J.H., \& Shenoy, S.S. 2001, ApJ, 547, 872

Willacy, K. 2004, ApJ, 600, L87

Zuckerman, B., Dyck, H.M., \& Claussen, M.J. 1986, ApJ, 304, 401 
Table 1. Model parameters for IRC $+10216^{\text {a }}$

\begin{tabular}{cc}
\hline \hline Parameter & Value \\
\hline Gas velocity field & $v(r)=14.5 \sqrt{1-0.95 \frac{R_{i}}{r}} \mathrm{~km} \mathrm{~s}^{-1}$ \\
Turbulent velocity & $v_{t}=0.65 \mathrm{~km} \mathrm{~s}^{-1}$ \\
$\mathrm{H}_{2}$ density & $n\left(\mathrm{H}_{2}\right)=\frac{3.11 \times 10^{7}}{r_{15}^{2}} \frac{14.5 \mathrm{kms}^{-1}}{v(r)} \mathrm{cm}^{-3}$ \\
Gas temperature & $T_{k}(r)=12\left(\frac{90}{r_{15}}\right)^{0.72} \mathrm{~K}$ if $r_{15}<110$ \\
Dust inner radius & $T_{k}(r)=10 \mathrm{~K}$ if $r_{15}>110$ \\
Dust outer radius & $R_{i}=4.5 \times 10^{14} \mathrm{~cm}$ \\
Stellar radius & $R_{o}=4.0 \times 10^{17} \mathrm{~cm}$ \\
Stellar temperature & $R_{*}=8.0 \times 10^{13} \mathrm{~cm}$ \\
Spectral index & $T_{*}=2050 \mathrm{~K}$ \\
& $\beta=1.3$ if $\lambda>20 \mu \mathrm{m}$ \\
& $\beta=1$ if $\lambda<20 \mu \mathrm{m}$ \\
\hline
\end{tabular}

${ }^{\mathrm{a}} r$ is the distance to the star in $\mathrm{cm}$, and $r_{15}=\frac{r}{10^{15} \mathrm{~cm}}$.

Table 2. Inner and outer radii for the $\mathrm{H}_{2} \mathrm{O}$ shell in different models, and derived o- $\mathrm{H}_{2} \mathrm{O}$ abundances

\begin{tabular}{cccc}
\hline \hline Model & $R_{\text {int }}(\mathrm{cm})$ & $R_{\text {out }}(\mathrm{cm})$ & $X\left(\mathrm{o}-\mathrm{H}_{2} \mathrm{O}\right)^{\mathrm{a}}$ \\
\hline$A$ & $4.5 \times 10^{14}$ & $4.0 \times 10^{17}$ & $4.1 \times 10^{-8}$ \\
$B$ & $2.1 \times 10^{15}$ & $4.0 \times 10^{17}$ & $5.4 \times 10^{-8}$ \\
$C$ & $4.3 \times 10^{16}$ & $4.0 \times 10^{17}$ & $5.1 \times 10^{-7}$ \\
\hline
\end{tabular}

${ }^{\mathrm{a}} \mathrm{O}-\mathrm{H}_{2} \mathrm{O}$ abundances are given relative to $\mathrm{H}_{2}$, and an ortho-to-para $\mathrm{H}_{2} \mathrm{O}$ abundance ratio of $3: 1$ is assumed in all models. 
Table 3. $\mathrm{H}_{2} \mathrm{O}$ transitions, frequencies, and adopted HSO-HIFI half-power beamwidths and sensitivities

\begin{tabular}{|c|c|c|c|}
\hline Transition & $\begin{array}{l}\text { Frequency } \\
\qquad(\mathrm{GHz})\end{array}$ & $\begin{array}{l}\text { HSO-HIFI beam }{ }^{\mathrm{a}} \\
\left(^{\prime \prime}\right)\end{array}$ & $\begin{array}{c}\text { Sensitivity }^{\mathrm{b}} \\
\left(10^{-22} \mathrm{~W} \mathrm{~cm}^{-2}\right)\end{array}$ \\
\hline 110101 & 556.9 & 41.0 & 2.2 \\
\hline 312303 & 1097.4 & 21.0 & 12 \\
\hline 312221 & 1153.1 & 18.5 & 29 \\
\hline 321312 & 1162.9 & 18.5 & 30 \\
\hline 523514 & 1410.6 & 14.5 & 45 \\
\hline 221212 & 1661.0 & 14.5 & 61 \\
\hline 212101 & 1669.9 & 14.5 & 62 \\
\hline 303212 & 1716.8 & 12.5 & 56 \\
\hline 532523 & 1867.7 & 12.5 & 65 \\
\hline 211202 & 752.0 & 29.0 & 4.1 \\
\hline 202111 & 987.9 & 21.0 & 9.2 \\
\hline 111000 & 1113.3 & 21.0 & 12 \\
\hline 422413 & 1207.6 & 18.5 & 32 \\
\hline 220211 & 1228.8 & 18.5 & 35 \\
\hline 413404 & 1602.2 & 14.5 & 55 \\
\hline 633624 & 1762.0 & 12.5 & 60 \\
\hline 624615 & 1794.8 & 12.5 & 64 \\
\hline
\end{tabular}

aAdopted from http://www.ipac.caltech.edu/Herschel/hifi/hifi.shtml

${ }^{\mathrm{b}}$ Calculated using HSPOT version 1.9.1; sensitivities correspond to $5 \sigma$ in $1 \mathrm{hr}$ with a spectral resolving power of $R=10^{4}$ 

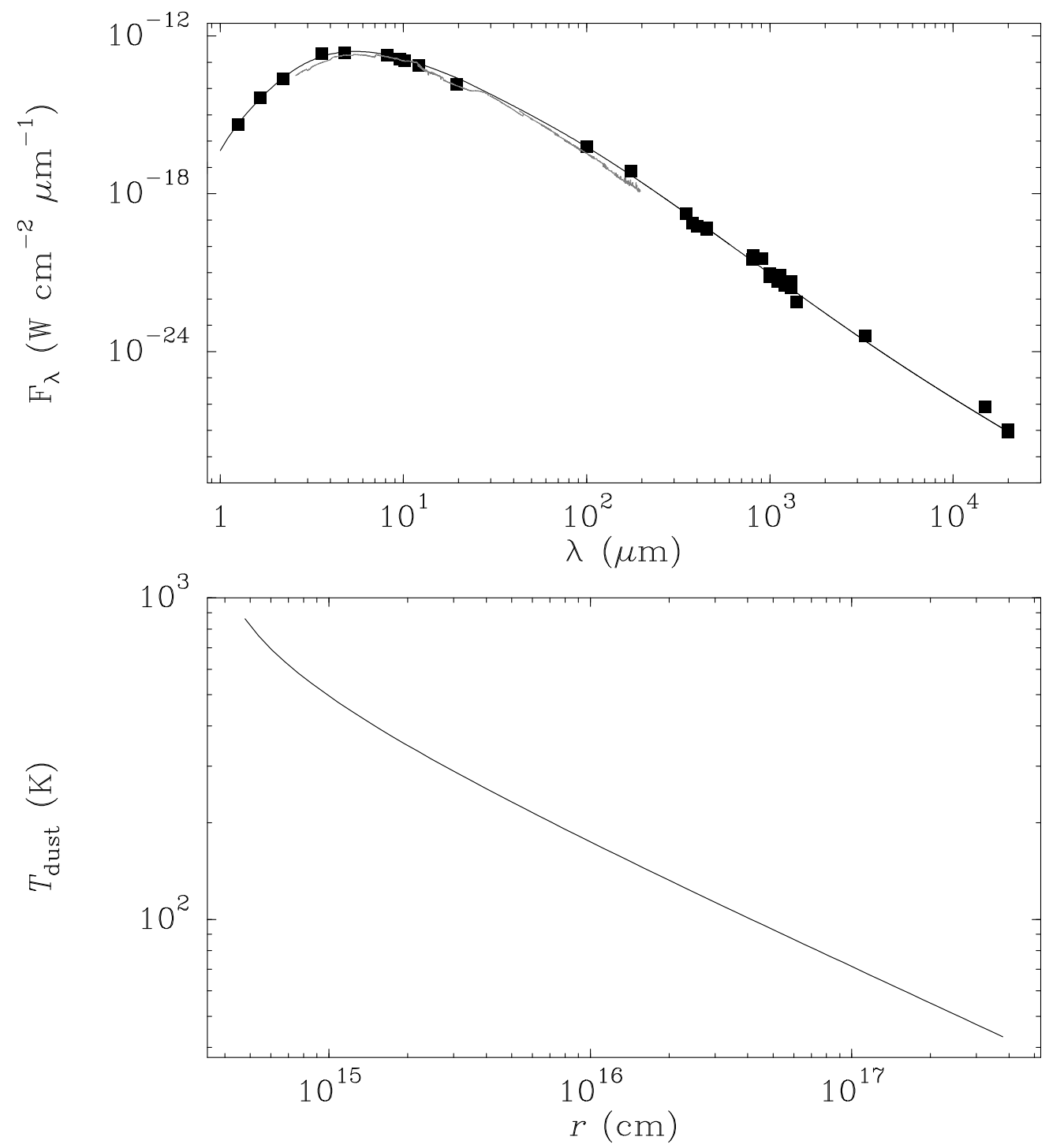

Fig. 1.- Top: Fit to the continuum emission from IRC+10216. Data points are taken from Bagnulo et al. (1995), and the grey line shows the ISO data. Bottom: Dust temperature profile. 


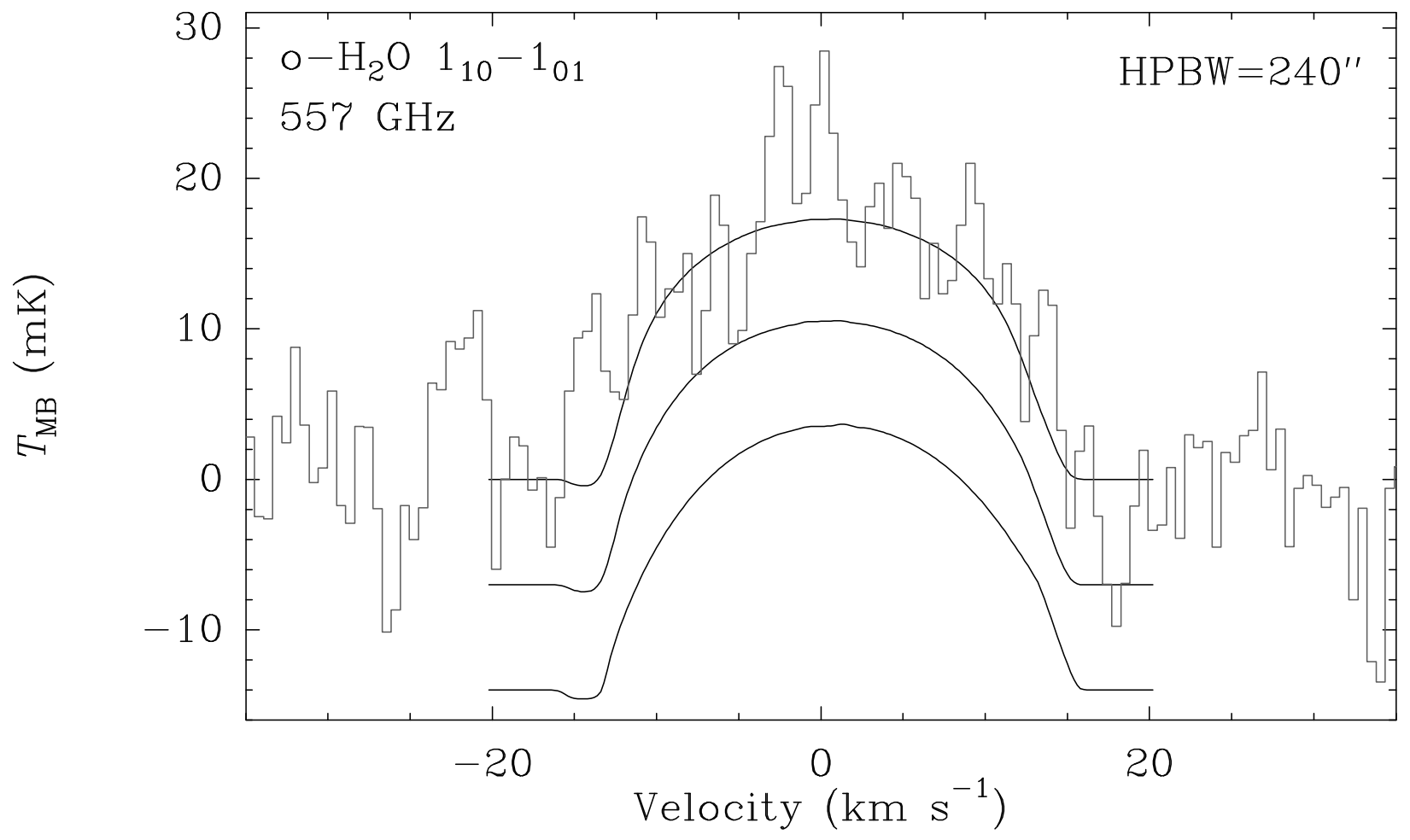

Fig. 2.- Comparison between the SWAS 110101 o- $\mathrm{H}_{2} \mathrm{O}$ spectrum (from Melnick et al. 2001) and the line profile obtained for models $A$ (upper), $B$ (middle), and $C$ (lower), with the SWAS beamsize. The spectra of models $B$ and $C$ have been shifted vertically for clarity. The required abundances of $\mathrm{o}-\mathrm{H}_{2} \mathrm{O}$ are given in Table 2 , 


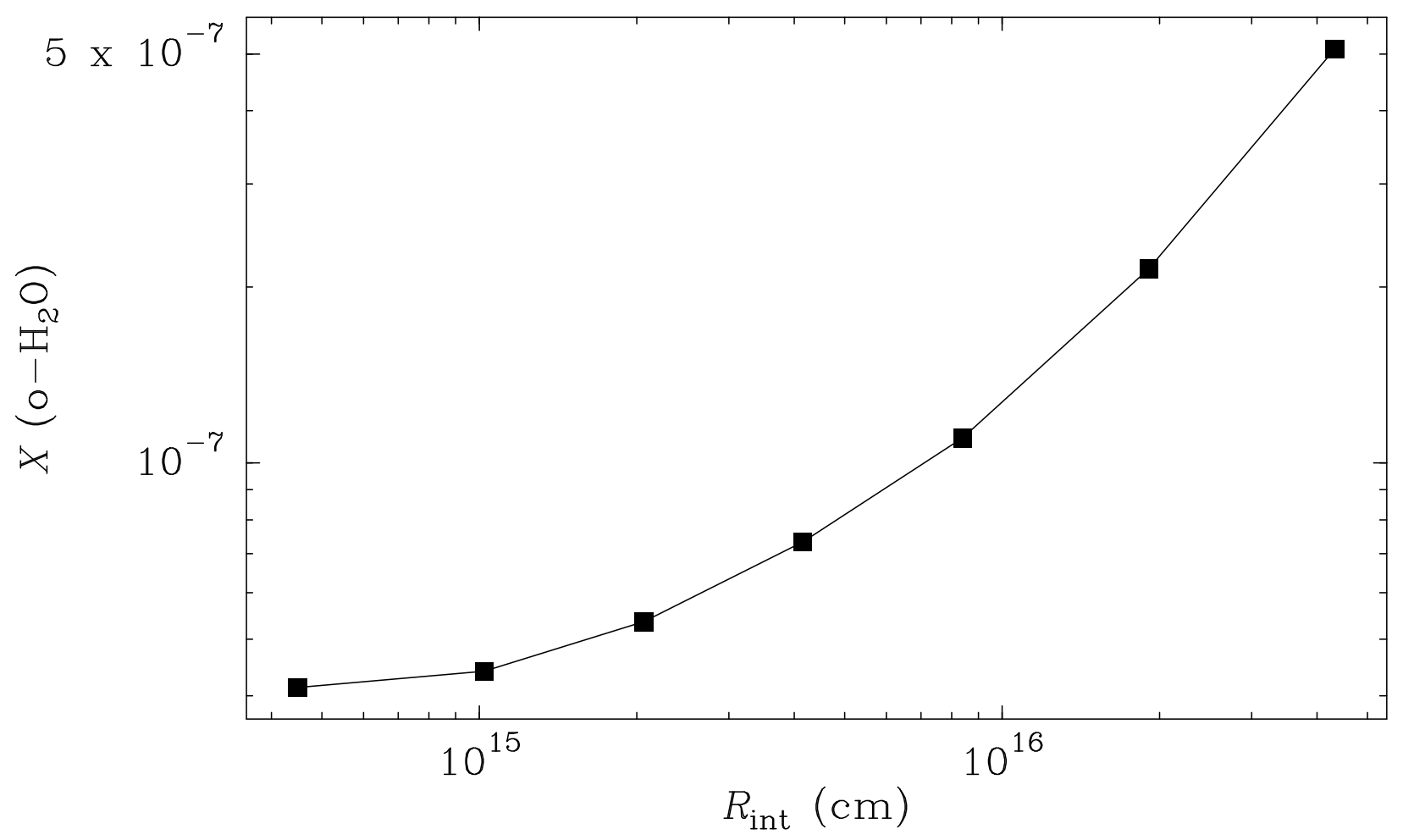

Fig. 3.- Ortho- $\mathrm{H}_{2} \mathrm{O}$ abundance, relative to $\mathrm{H}_{2}$, required to account for the observed 557 $\mathrm{GHz} \mathrm{H}_{2} \mathrm{O}$ emission in IRC+10216, as a function of the assumed inner radius of the $\mathrm{H}_{2} \mathrm{O}$ shell. 


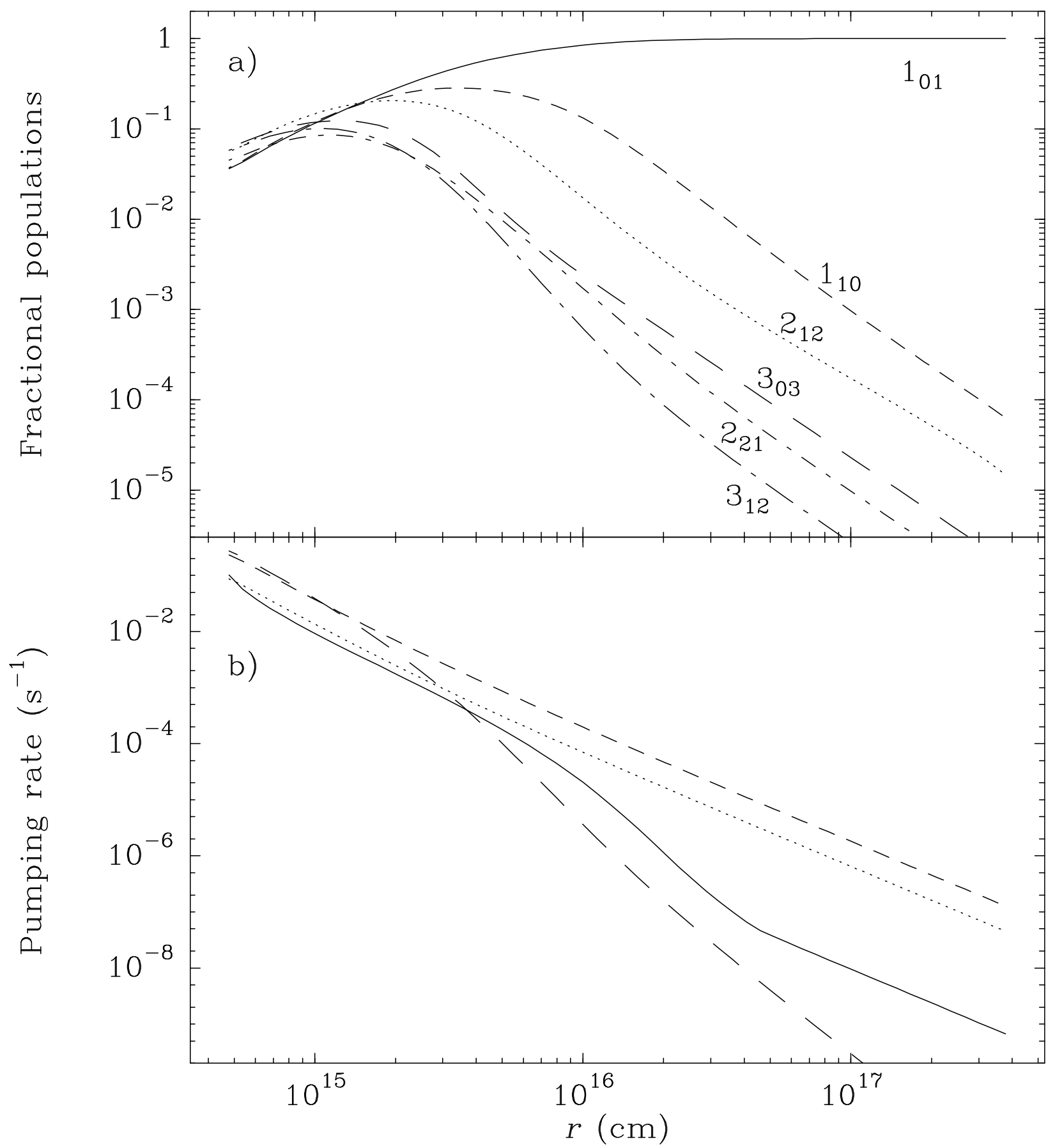

Fig. 4.- a) Fractional populations of the 6 lowest energy levels of ortho- $\mathrm{H}_{2} \mathrm{O}$ in model $A$; b) collisional and radiative pumping rates, per molecule in the $1_{01}$ level, for the $1_{10}$ level in model $A$. Solid line: collisional pumping, which is computed from the $1_{01} \rightarrow 1_{10}$ collisional rate; dashed line: radiative rate through the path $1_{01} \rightarrow \nu_{2} 1_{10} \rightarrow 2_{21} \rightarrow 1_{10}$; dotted line: radiative rate through the path $1_{01} \rightarrow \nu_{2} 2_{12} \rightarrow 2_{21} \rightarrow 1_{10}$; long-dashed line: radiative rate through the path $2_{12} \rightarrow \nu_{2} 1_{01} \rightarrow 1_{10}$. 


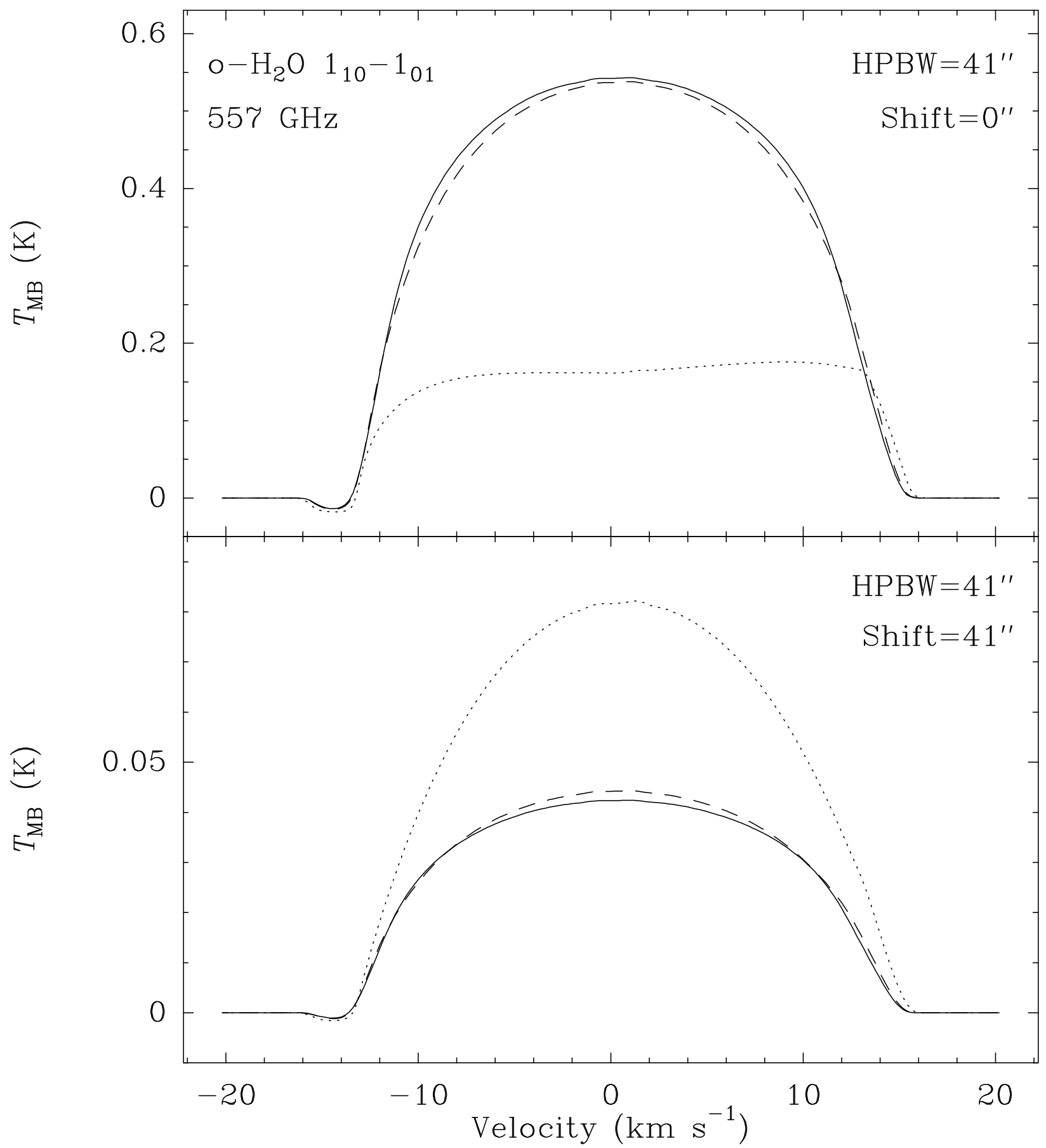

Fig. 5.- Predicted line profile of the o- $\mathrm{H}_{2} \mathrm{O} 110101$ line with the HSO-HIFI beam, and the telescope axis pointing toward the source center (upper) and toward a position shifted 41" from the source center (lower). Model $A$ : solid line; $B$ : dashed line; $C$ : dotted line. 


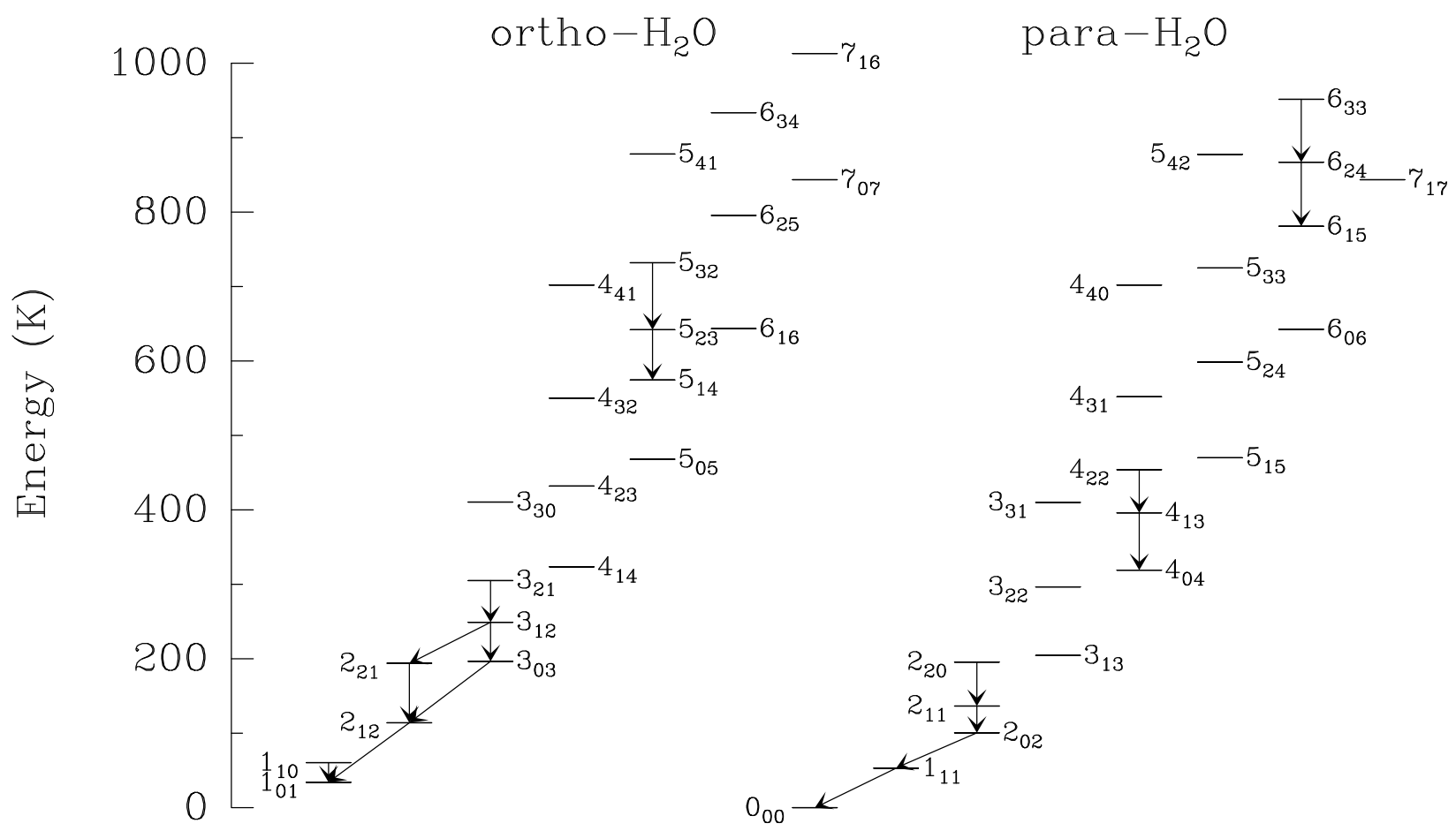

Fig. 6.- Energy level diagram of ortho and para- $\mathrm{H}_{2} \mathrm{O}$. Arrows indicate the transitions whose predicted line fluxes and profiles are shown in Figs. 7 and 8 . 

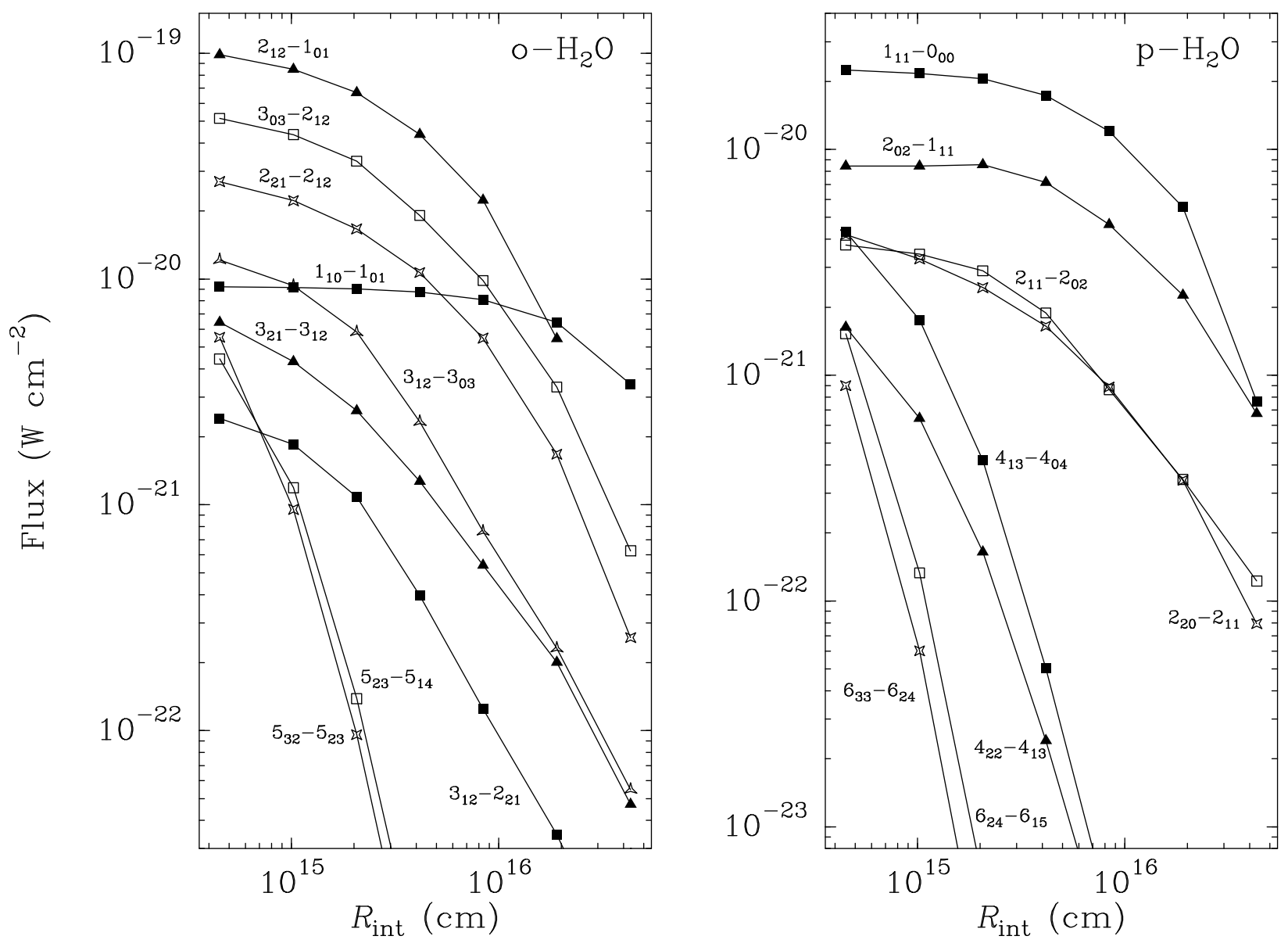

Fig. 7.- Predicted $\mathrm{H}_{2} \mathrm{O}$ line fluxes observable by HSO-HIFI as a function of the inner radius of the $\mathrm{H}_{2} \mathrm{O}$ shell. Adopted beamwidths are listed in Table 3 . 

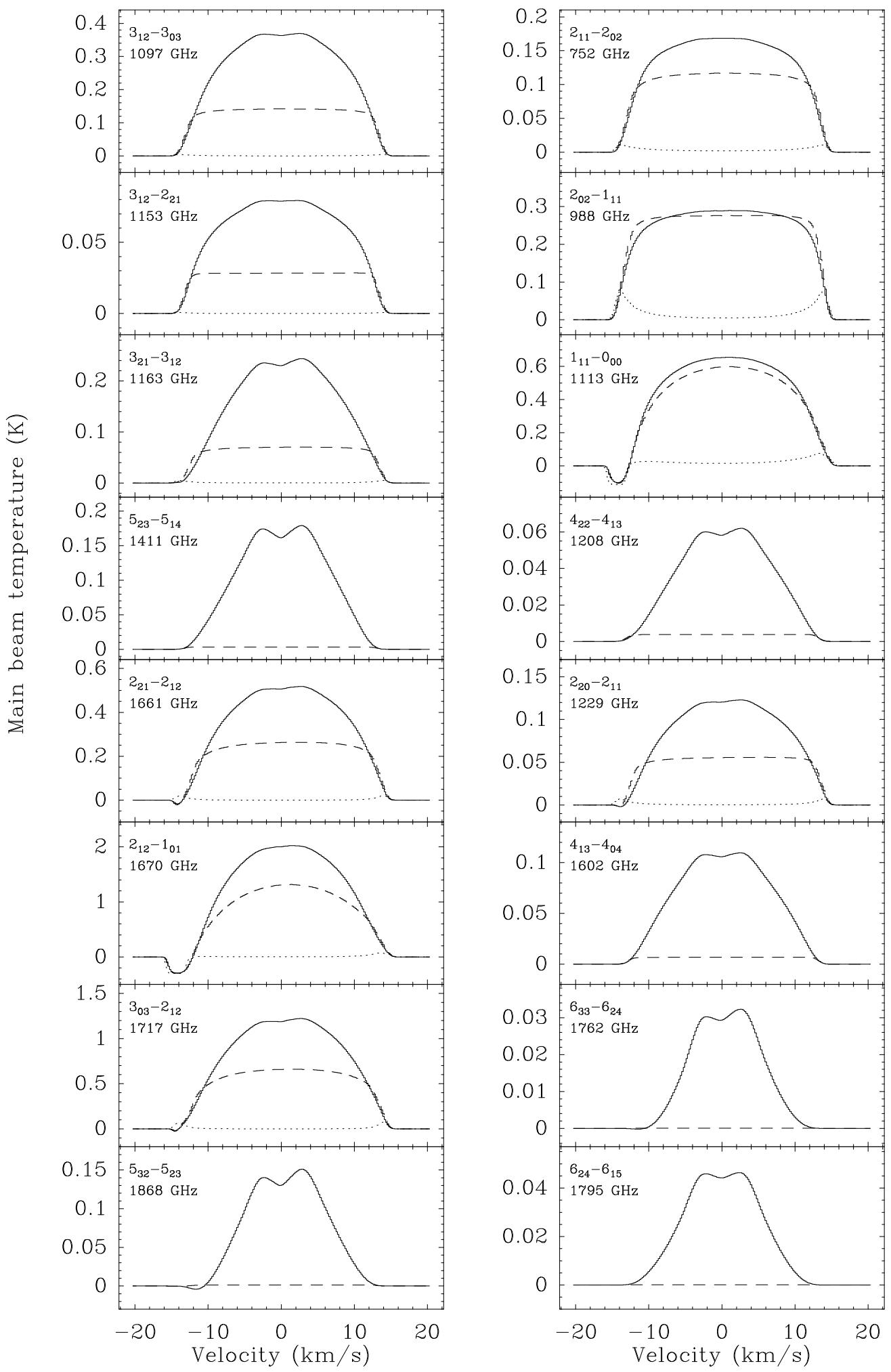

Fig. 8.- Predicted $\mathrm{H}_{2} \mathrm{O}$ line profiles observable by HSO-HIFI, for models $A$ (solid), $B$ (dashed), and $C$ (dotted). Adopted beamwidths are listed in Table 3 . 


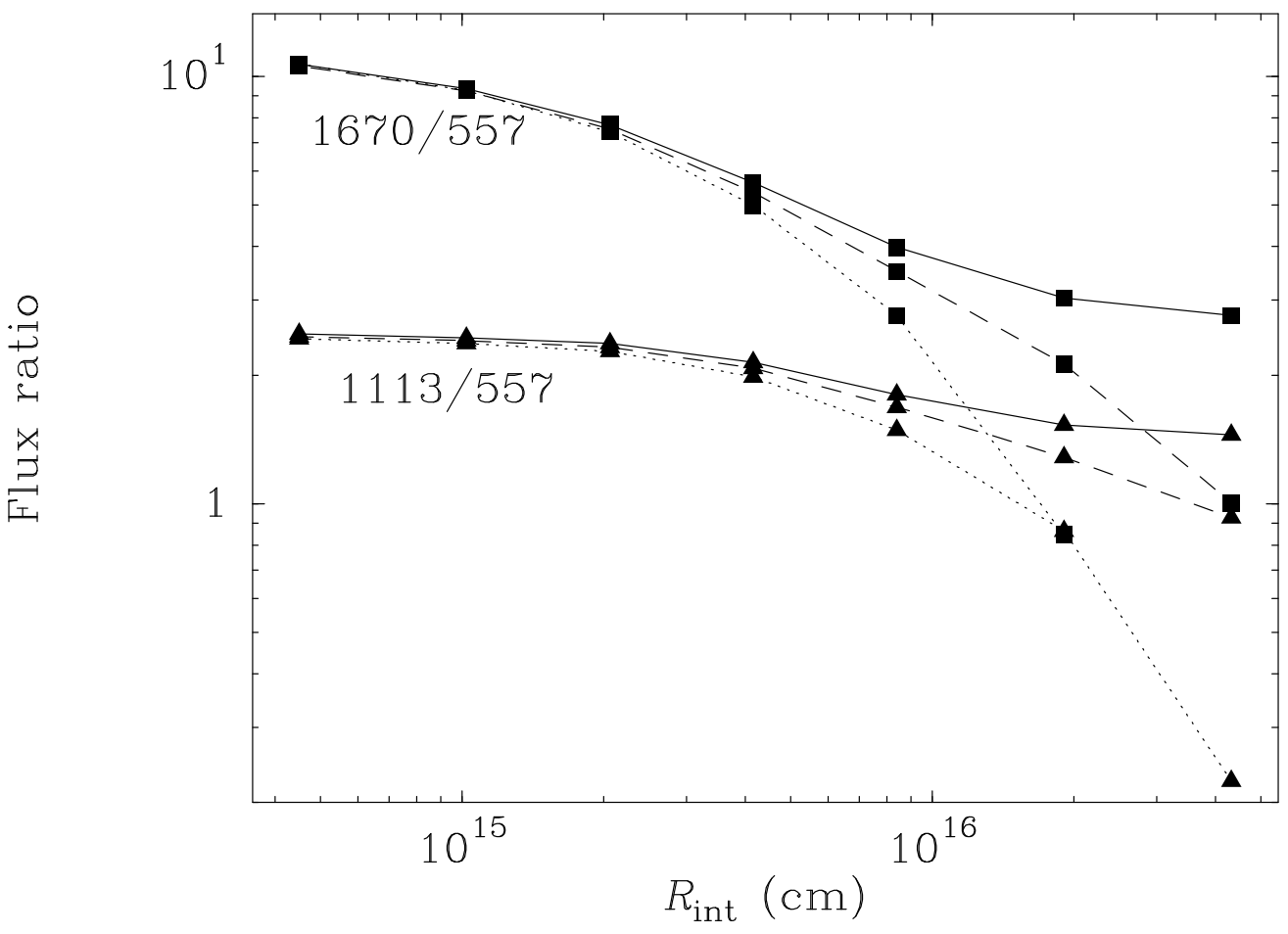

Fig. $\quad$ 9.- Calculated $F(212101) / F(110101)$ (labelled 1670/557, squares) and $F(111000) / F(110101)$ (labelled $1113 / 557$, triangles) vs. $R_{\text {int }}$ for the IRC +10216 model source, but located at a distance of $D>2 \mathrm{kpc}$ (solid lines), $D=0.5 \mathrm{kpc}$ (dashed lines), and $D=0.17 \mathrm{kpc}$ (dotted lines). 


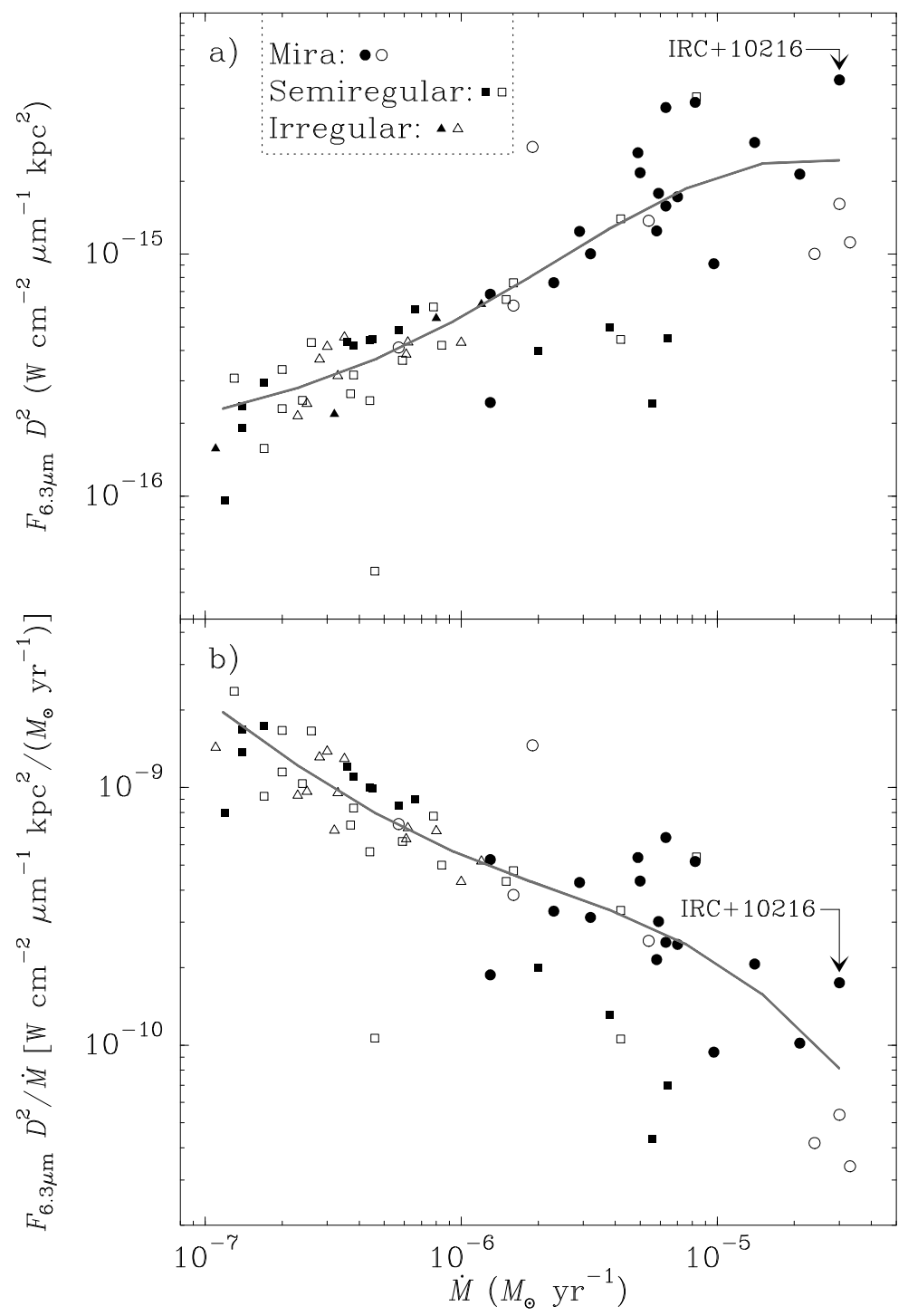

Fig. 10.- a) Continuum flux densities at $6.3 \mu \mathrm{m}$, corrected for the distance to the sources, versus the mass loss rate for a sample of C-rich AGB stars. Filled symbols indicate sources for which the $6.3 \mu \mathrm{m}$ flux has been directly measured from ISO/SWS spectra (Sloan et al. 2003), and opened symbols indicate sources for which the $6.3 \mu \mathrm{m}$ flux has been estimated from the available $8.8 \mu \mathrm{m}$ flux density (see text for details). The grey line shows the fit to the general trend achieved with our dust models. b) Same as in a) but divided by the mass loss rate. The position of $\mathrm{IRC}+10216$ is indicated in both diagrams. 

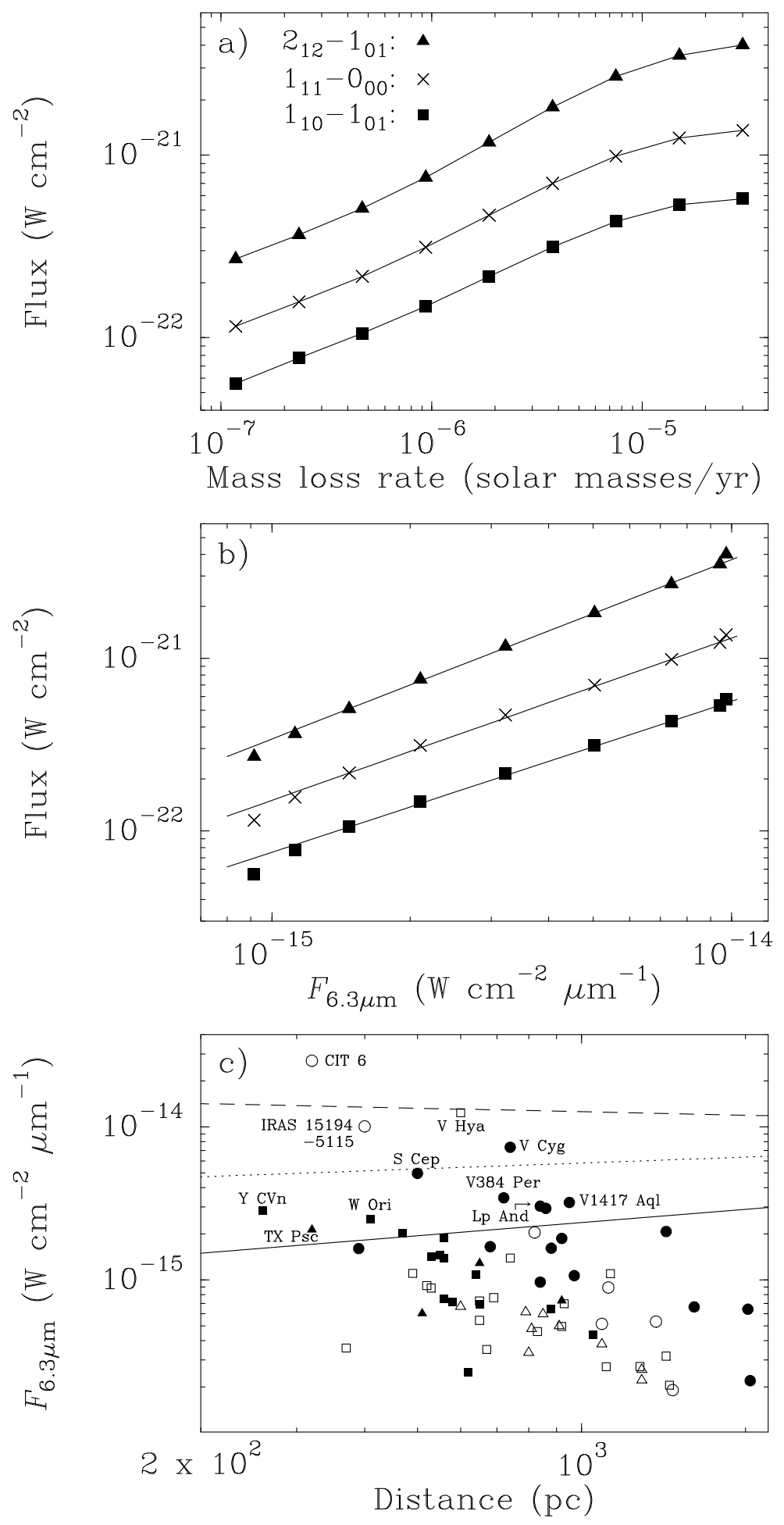

Fig. 11. - Calculated $\mathrm{H}_{2} \mathrm{O}$ line fluxes vs. $\dot{M}$ (a), and vs. the $6.3 \mu \mathrm{m}$ continuum flux (b), where the lines are fits of the form $F_{\text {line }} \propto F_{6.3 \mu \mathrm{m}}^{b}$; results are given for a source at $D=0.5$ kpc. c) Observed continuum fluxes at $6.3 \mu \mathrm{m}$ of C-rich AGB stars in function of the distance (see Fig. 10 for the meaning of symbols). The lines show the $6.3 \mu \mathrm{m}$ continuum flux required to detect the ground-state lines in 1.5 hours of observing time with HSO-HIFI, if the $\mathrm{H}_{2} \mathrm{O}$ outflow rate is the same as that in IRC+10216 (solid: 110101; dotted: 111000; dashed: 212101). Some of the stars that would be detectable in such a case in the $110101 \mathrm{o}-\mathrm{H}_{2} \mathrm{O}$ line 


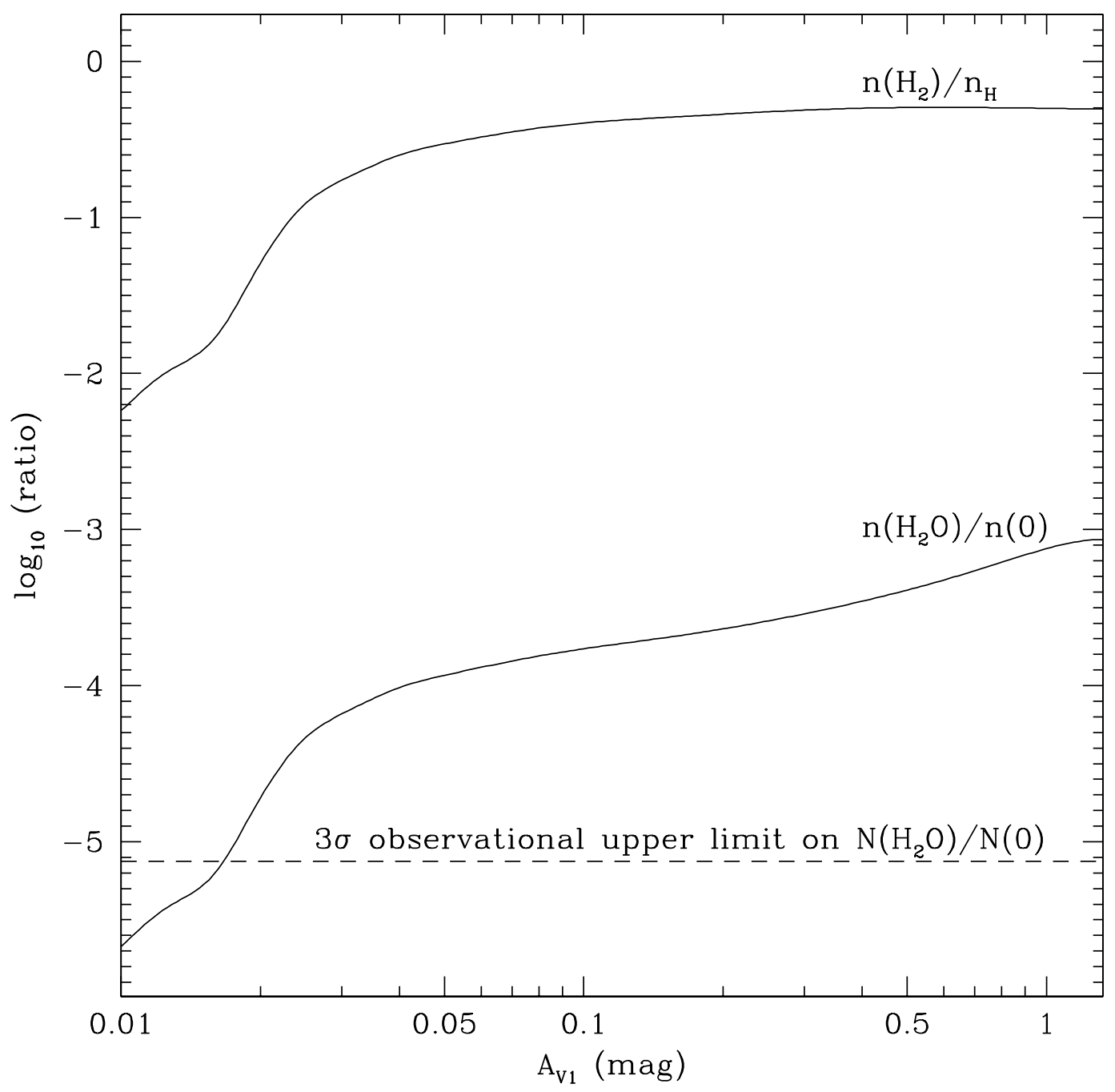

Fig. 12.- Predicted $n\left(\mathrm{H}_{2}\right) / n_{\mathrm{H}}$ ratio for the best-fit Spaans et al. (1998) model, together with the $n\left(\mathrm{H}_{2} \mathrm{O}\right) / n(\mathrm{O})$ ratio that would result if $k_{r a}$ were $10^{-15} \mathrm{~cm}^{3} \mathrm{~s}^{-1}$. The dashed line shows the $3 \sigma$ upper limit on the observed $N\left(\mathrm{H}_{2} \mathrm{O}\right) / N(\mathrm{O})$ ratio. 